\title{
Behavioral Learning Equilibria
}

\author{
Cars Hommes*, Mei Zhu \\ CeNDEF, School of Economics, University of Amsterdam \\ Roetersstraat 11, 1018 WB Amsterdam, Netherlands
}

August 2011

\begin{abstract}
We propose behavioral learning equilibria as a plausible explanation of coordination of individual expectations and aggregate phenomena such as excess volatility in stock prices and high persistence in inflation. Boundedly rational agents use a simple univariate linear forecasting rule and in equilibrium correctly forecast the unconditional sample mean and first-order sample autocorrelation. In the long run, agents thus learn the best univariate linear forecasting rule, without fully recognizing the structure of the economy. The simplicity of our behavioral learning equilibria makes coordination of individual expectations on such an aggregate outcome more likely. In a first application, an asset pricing model with $\mathrm{AR}(1)$ dividends, a unique stochastic consistent expectations equilibrium (SCEE) exists characterized by high persistence and excess volatility, and it is globally stable under learning. In a second application, the New Keynesian Phillips curve, multiple equilibria co-exist and learning exhibits path dependence and inflation may switch between low and high persistence regimes.

Keywords: Bounded rationality; Stochastic consistent expectations equilibrium; Adaptive learning; Excess volatility; Inflation persistence JEL classification: E30; C62; D83; D84
\end{abstract}

${ }^{*}$ Corresponding author. Tel.: +31 20525 4246; fax: +31 205254349 .

E-mail address: C.H.Hommes@uva.nl. 


\section{Introduction}

Expectation feedback plays a crucial role in economics and finance. Since the introduction by Muth (1961), and its application in macroeconomics by Lucas (1972), the Rational Expectation Hypothesis (REH) has become the predominant paradigm. A Rational Expectation Equilibrium (REE) is in fact a fixed point of an expectation feedback system. Typically it is assumed that rational agents perfectly know the correctly specified market equilibrium equations as well as their parameter values.

Despite its popularity, the REH has been criticized for its highly demanding and unrealistic information requirements. Adaptive learning models have been proposed as an alternative to rational expectations (Marcet and Sargent, 1989; Bullard, 1994; see Sargent (1993, 1999) and Evans and Honkapohja (2001) for extensive surveys). In contrast to rational expectations, adaptive learning models assume that agents do not have perfect knowledge about market equilibrium equations, but agents are assumed to have some belief, the perceived law of motion, about the actual law of motion; the corresponding parameters are not known, but are estimated by adaptive learning based on available observations. The implied actual law of motion under adaptive learning is thus a timevarying self referential system, depending on the perceived law of motion. Under this framework, a rational expectations equilibrium is simply a situation in which the implied law of motion exactly coincides with the perceived law of motion, and adaptive learning may converge to such a rational expectations equilibrium. In other words, convergence of adaptive learning to a rational expectations equilibrium can occur when the perceived law of motion is correctly specified.

In general a perceived law of motion will be misspecified. White (1994) argues that an economic model or a probability model is only a more or less crude approximation to whatever might be the "true" relationships among the observed data and consequently it is necessary to view economic and/or probability models as misspecified to some greater or lesser degree. Sargent (1991) first develops a notion of equilibrium as a fixed point of an operator that maps the perceived law of motion (a vector ARMA process) into a statistically optimal estimator of the actual law of motion. This may be viewed as an early example of a Restricted Perceptions Equilibrium (RPE), as defined by Evans and Honkapohja (2001), formalizing the idea that agents have misspecified beliefs, but within the context of their forecasting model they are unable to detect their misspecification. Branch (2006) gives an excellent survey and argues that the RPE is a natural alternative 
to rational expectation equilibrium because it is to some extent consistent with Muth's original hypothesis of REE while allowing for bounded rationality by restricting the class of the perceived law of motion.

The main contribution of our paper is to develop a behavioral learning equilibrium concept, where agents try to learn a simple but misspecified forecasting rule. Our equilibrium concept - Stochastic Consistent Expectations Equilibrium (SCEE) - may be viewed as the simplest RPE and therefore it seems more likely as a description of aggregate behavior, because a large population of indiviudal agents may coordinate their expectations more easily and learn such a simple behavioral equilibrium. The actual law of motion (ALM) of the economy is a two (or higher) dimensional linear stochastic system. Agents are forecasting one variable - say the price - of the economy using a simple univariate $\operatorname{AR}(1)$ forecasting rule. In a SCEE the mean and the first-order autocorrelation of realized prices in the economy coincide with the corresponding mean and first-order autocorrelation of agents' $\mathrm{AR}(1)$ perceived law of motion (PLM). In addition, a simple adaptive learning scheme - Sample Autocorrelation Leaning (SAC-learning) - with an intuitive behavioral interpretation, enforces convergence to the (stable) SCEE.

We illustrate our behavioral learning equilibrium concept in two standard applications. In the first - an asset pricing model with an exogenous stochastic dividend process - the SCEE is unique and the SAC-learning scheme always converges to the SCEE. The SCEE is characterized by highly persistent prices (close to unit root) and excess volatility with asset prices volatility more than doubled compared to REE. In the second application - a New Keynesian Philips curve (NKPC) - with an exogenous AR(1) process for the output gap and an independent and identically distributed (i.i.d.) stochastic shock to inflation - multiple stable SCEE may co-exist. In particular, for empirically plausible parameter values a SCEE with highly persistent inflation exists, matching the stylized facts of US-inflation data.

\section{Related literature}

Our behavioral equilibrium is closely related to the Consistent Expectations Equilibrium (CEE) introduced by Hommes and Sorger (1998), where agents believe that prices follow a linear AR(1) stochastic process, whereas the implied actual law of motion is a deterministic chaotic nonlinear process. Along a CEE, price realizations have the same sample mean and sample autocorrelation coefficients as the $\mathrm{AR}(1)$ perceived law of mo- 
tion. A CEE is another early example of a RPE and may be seen as an "approximate rational expectations equilibrium", in which the misspecified perceived law of motion is the best linear approximation within the class of perceived laws of motion of the actual (unknown) nonlinear law of motion. Hommes and Rosser (2001) investigate CEE in an optimal fishery management model and used numerical simulations to study adaptive learning of $\mathrm{CEE}$ in the presence of dynamic noise. The adaptive learning scheme used here is SAC-learning, where the parameters of the $\mathrm{AR}(1)$ forecasting rule are updated based on the observed sample average and first-order sample autocorrelation. Sögner and Mitlöhner (2002) apply the CEE concept to a standard asset pricing model with independent and identically distributed (i.i.d.) dividends and showed that the unique CEE coincides with the REE. As we will see in the current paper, introducing autocorrelations in the stochastic dividend process will lead to learning equilibrium different from REE. Tuinstra (2003) analyzes first-order consistent expectations equilibria numerically in a deterministic overlapping generations (OLG) model. Hommes et al (2004) generalize the notion of CEE to nonlinear stochastic dynamic economic models, introducing the concept of stochastic consistent expectations equilibrium (SCEE). In a SCEE, agents' perceptions about endogenous variables are consistent with the actual realizations of these variables in the sense that the unconditional mean and autocorrelations of the unknown nonlinear stochastic process, which describes the actual behavior of the economy, coincide with the unconditional mean and autocorrelations of the AR(1) process agents believe in. They applied this concept to an OLG model and studied the existence of SCEE and its relationship to sample autocorrelation learning (SAC-learning) based on numerical simulations.

Showing theoretically existence of SCEE and its relationship to adaptive learning has proven to be technically difficult, while convergence of SAC-learning has been studied only by numerical simulations. The principle technical difficulty here is to calculate autocorrelation coefficients, prove existence of fixed points in a nonlinear system and analyze the relationship between SCEE and sample autocorrelation learning. Branch and McGough (2005) obtain existence results on first-order SCEE theoretically and analyze the stability of SCEE under real-time learning numerically in a stochastic non-linear self-referential model where expectations are based on an AR(1) process. Lansing (2009) considers a special class of SCEE in the New Keynesian Philips curve, where the value of the Kalman gain parameter in agents' forecast rule is pinned down using the observed autocorrelation of inflation changes. Lansing (2010) studies a Lucas-type asset pricing model and found numerically a near-rational restricted perceptions equilibrium, for which the covariance of 
an underparameterized (one parameter) PLM coincides with the covariance of an approximate ALM. Bullard et al. $(2008,2010)$ add judgment into agents' forecasts and use the concept of SCEE to provide a related interesting concept of exuberance equilibria. They study the resulting dynamics in the New Keynesian model and a standard asset pricing model, respectively, where the driving variables are white noises (no autocorrelations).

The current paper studies the existence of SCEE and its stability under SAC-learning in two standard applications: an asset pricing model and the New Keynesian Philips curve. In both applications the driving variables (dividends or real marginal costs) are assumed to follow $\mathrm{AR}(1)$ processes. More specifically, while the perceived law of motion agents believe in is an $\mathrm{AR}(1)$ process with white noise, the true process of economy is not an $\mathrm{AR}(1)$ process but a linear stochastic process driven by an exogenous autocorrelated process. In addition to the conceptual contribution of introducing a behavioral learning equilibrium, our paper makes two methodological contributions. First, we prove existence of SCEE under general conditions in a misspecified framework, where prices (inflation) have the same mean as REE. Second, we present the first proof that the SAC-learning converges to stable SCEE and provide simple and intuitive stability conditions. SCEE thus represents a fixed point of learning dynamics under misspecification. Moreover, we provide interesting results in our two applications. In the asset pricing model, we show that the SCEE is unique and (globally) stable and characterized by market prices fluctuating around fundamental prices and exhibiting stronger serial autocorrelations and higher volatility than the REE for plausible parameters. In the New Keynesian Philips curve, we show that multiple SCEE may exist. In particular, for a large set of plausible parameters a SCEE exists with highly persistent inflation. Coordination on a behavioral learning equilibrium may thus explain high persistence in inflation (Milani, 2007).

Some other related literature, for example Timmermann (1993, 1996), Bullard and Duffy (2001), Guidolin and Timmermann (2007) and Bullard et al. (2010), shows the effects of learning on asset returns from different perspectives. Timmermann $(1993,1996)$ shows that learning helps to explain excess volatility and predictability of stock returns in the similar present value asset pricing model. In Timmermann (1993, 1996), the perceived law of motion is correctly specified but the related parameters are estimated by adaptive learning, and in the long run learning converges to REE. Bullard and Duffy (2001) introduce adaptive learning into a general-equilibrium life-cycle economy with capital accumulation and show that in contrast to perfect-foresight dynamics, the system under least-squares learning possesses equilibria that are characterized by persistent 
excess volatility in returns to capital. Guidolin and Timmermann (2007) characterize equilibrium asset prices under adaptive, rational and Bayesian learning schemes in a model where dividends evolve on a binomial lattice and find that learning introduces serial correlation and volatility clustering in stock returns. Bullard et al. (2010) construct a simple asset pricing example with constant known dividends and i.i.d. asset supply and find that exuberance equilibria, when they exist, can be extremely volatile relative to fundamental equilibria. An important conceptual difference with these references is our behavioral interpretation of the SCEE as what is perhaps the simplest example of RPE. A behavioral SCEE together with an intuitive SAC-learning scheme may explain coordination of individual expectations on (almost) self-fulfilling equilibria.

The paper is organized as follows. Section 2 introduces the main concepts, i.e. firstorder SCEE and sample autocorrelation learning in a general framework. Section 3 studies existence and stability under SAC-learning theoretically as well as numerically in a standard asset pricing model. Section 4 presents a second application, the New Keynesian Philips curve, and shows existence of multiple SCEE and the relationship to SAC-learning theoretically and numerically. Finally, section 5 concludes.

\section{Preliminary concepts}

This section briefly introduces the main concepts. Suppose that the law of motion of an economic system is given by the stochastic difference equation

$$
x_{t}=f\left(x_{t+1}^{e}, y_{t}, u_{t}\right)
$$

where $x_{t}$ is the state of the system (e.g. asset price or inflation) at date $t$ and $x_{t+1}^{e}$ is the expected value of $x$ at date $t+1$. This denotation highlights that expectations may not be rational. Here $f$ is a continuous function, $\left\{u_{t}\right\}$ is an i.i.d. noise process with mean zero and finite absolute moments ${ }^{1}$, where the variance is denoted by $\sigma_{u}^{2}$, and $y_{t}$ is a driving variable (e.g. dividends or the output gap), assumed to follow an exogenous stochastic $\mathrm{AR}(1)$ process

$$
y_{t}=a+\rho y_{t-1}+\varepsilon_{t}, \quad 0 \leq \rho<1,
$$

where $\left\{\varepsilon_{t}\right\}$ is another i.i.d. noise process with mean zero and finite absolute moments, with variance $\sigma_{\varepsilon}^{2}$, and uncorrelated with $\left\{u_{t}\right\}$. The mean of the stationary process $y_{t}$ is

\footnotetext{
${ }^{1}$ The condition on finite absolute moments is required to obtain convergence results under SAClearning.
} 
$\bar{y}=\frac{a}{1-\rho}$, the variance is $\sigma_{y}^{2}=\frac{\sigma_{\varepsilon}^{2}}{1-\rho^{2}}$ and the $k$ th-order autocorrelation coefficient of $y_{t}$ is $\rho^{k}$, see for example, Hamilton (1994).

Agents are boundedly rational and do not know the exact form of the actual law of motion in (2.1). We assume that, in order to forecast $x_{t+1}$, agents only use past observations $x_{t-1}, x_{t-2}, \cdots$, etc. Hence agents do not recognize that $x_{t}$ is driven by an exogenous stochastic process $y_{t}$. Instead agents believe that the economic variable $x_{t}$ follows a simple linear stochastic process. More specifically, agents' perceived law of motion (PLM) is an AR(1) process, as in Hommes et al. (2004) and Branch and McGough (2005), i.e.

$$
x_{t}=\alpha+\beta\left(x_{t-1}-\alpha\right)+\delta_{t}
$$

where $\alpha$ and $\beta$ are real numbers with $\beta \in(-1,1)$ and $\left\{\delta_{t}\right\}$ is a white noise process; $\alpha$ is the unconditional mean of $x_{t}$ while $\beta$ is the first-order autocorrelation coefficient. Given the perceived law of motion (2.3), the 2-period ahead forecasting rule for $x_{t+1}$ that minimizes the mean-squared forecasting error is

$$
x_{t+1}^{e}=\alpha+\beta^{2}\left(x_{t-1}-\alpha\right)
$$

Combining the expectations (2.4) and the law of motion of the economy (2.1), we obtain the implied actual law of motion (ALM)

$$
x_{t}=f\left(\alpha+\beta^{2}\left(x_{t-1}-\alpha\right), y_{t}, u_{t}\right) \text {, }
$$

with $y_{t}$ an $\mathrm{AR}(1)$ process as in (2.2).

\section{Stochastic Consistent Expectations Equilibrium (SCEE)}

We are now ready to recall the definition of stochastic consistent expectations equilibrium (SCEE). Following Hommes et al. $(2004)^{2}$, the concept of first-order SCEE is defined as follows.

Definition 2.1 A triple $(\mu, \alpha, \beta)$, where $\mu$ is a probability measure and $\alpha$ and $\beta$ are real numbers with $\beta \in(-1,1)$, is called a first-order stochastic consistent expectations equilibrium (SCEE) if the three conditions are satisfied:

S1 The probability measure $\mu$ is a nondegenerate invariant measure for the stochastic difference equation (2.5);

\footnotetext{
${ }^{2}$ In Hommes et al. (2004), the actual law of motion is $x_{t}=f\left(x_{t+1}^{e}, u_{t}\right)$, without the driving variable $y_{t}$. However, the definitions of SCEE and SAC-learning can still be applied here.
} 
S2 The stationary stochastic process defined by (2.5) with the invariant measure $\mu$ has unconditional mean $\alpha$, that is, $E_{\mu}(x)=\int x d \mu(x)=\alpha$;

S3 The stationary stochastic process defined by (2.5) with the invariant measure $\mu$ has unconditional first-order autocorrelation coefficient $\beta$.

That is to say, a first-order SCEE is characterized by the fact that both the unconditional mean and the unconditional first-order autocorrelation coefficient generated by the actual (unknown) stochastic process (2.5) coincide with the corresponding statistics for the perceived linear AR(1) process (2.3). This means that in a first-order SCEE agents correctly perceive the mean and the first-order autocorrelation (persistence) of economic variables although they do not correctly specify their model of the economy.

Our SCEE concept may be viewed as the simplest example of a RPE. It should be stressed that the SCEE has an intuitive behavioral interpretation. In a SCEE agents use a linear forecasting rule with two parameters, the mean $\alpha$ and the first-order autocorrelation $\beta$. Both can be observed from past observations by inferring the average price (or inflation level) and the (first-order) persistence of the time series. For example, $\beta=0.5$ means that, on average, prices mean revert toward their long-run mean by 50 percent. These observations could be made approximately and simply by observing the time series of aggregate variables. It is interesting to note that in learning-to-forecast laboratory experiments with human subjects, for many subjects forecasting behavior can indeed be described by simple rules, such as a simple AR(1) rule, see for example, Hommes et al. (2005), Adam (2007), Heemeijer et al. (2005), Hommes (2011).

Finally, we note that in a first-order SCEE, the orthogonality condition imposed by Restricted Perceptions Equilibrium (RPE)

$$
E x_{t-1}\left[x_{t}-\alpha-\beta\left(x_{t-1}-\alpha\right)\right]=E\left(x_{t-1}-\alpha\right)\left[x_{t}-\alpha-\beta\left(x_{t-1}-\alpha\right)\right]=0
$$

is satisfied. The orthogonality condition shows that agents can not detect the correlation between their forecasting errors and the agent's perceived model, see Branch (2006). The first-order SCEE is a RPE where agents have their model incorrect; but within the context of their forecasting model agents are unable to detect their misspecification.

\section{Sample autocorrelation learning}

In the above definition of first-order SCEE, agents' beliefs are described by the linear forecasting rule (2.4) with fixed parameters $\alpha$ and $\beta$. However, the parameters $\alpha$ and 
$\beta$ are usually unknown. In the adaptive learning literature, it is common to assume that agents behave like econometricians using time series observations to estimate the parameters as additional observations become available. Following Hommes and Sorger (1998), we assume that agents use sample autocorrelation learning (SAC-learning) to learn the parameters $\alpha$ and $\beta$. That is, for any finite set of observations $\left\{x_{0}, x_{1}, \cdots, x_{t}\right\}$, the sample average is given by

$$
\alpha_{t}=\frac{1}{t+1} \sum_{i=0}^{t} x_{i},
$$

and the first-order sample autocorrelation coefficient is given by

$$
\beta_{t}=\frac{\sum_{i=0}^{t-1}\left(x_{i}-\alpha_{t}\right)\left(x_{i+1}-\alpha_{t}\right)}{\sum_{i=0}^{t}\left(x_{i}-\alpha_{t}\right)^{2}} .
$$

Hence $\alpha_{t}$ and $\beta_{t}$ are updated over time as new information arrives.

Adaptive learning is sometimes referred to as statistical learning, because agents act as statisticians or econometricians and use a statistical procedure such as OLS to estimate and update parameters over time. SAC-learning may be viewed as another statistical learning procedure. We would like to stress however that SAC-learning has a simple behavioral interpretation that agents simply infer the sample average and persistence (i.e. first-order autocorrelation) from time series observations. We focus on the entire sample average for $\alpha_{t}$ in (2.6) and sample first-order autocorrelation for $\beta_{t}$ in (2.7) over the entire time-horizon, but one could also restrict the learning to the last $T$ observations with $T$ relatively small (e.g., $T=100$ or even smaller). It is an easy and natural way for agents, especially those without professional training, to estimate mean and first-order autocorrelation directly based on data instead of some complicated statistical techniques.

Define

$$
R_{t}=\frac{1}{t+1} \sum_{i=0}^{t}\left(x_{i}-\alpha_{t}\right)^{2},
$$

then the SAC-learning is equivalent to the following recursive dynamical system (see Appendix A).

$$
\left\{\begin{aligned}
\alpha_{t} & =\alpha_{t-1}+\frac{1}{t+1}\left(x_{t}-\alpha_{t-1}\right), \\
\beta_{t} & =\beta_{t-1}+\frac{1}{t+1} R_{t}^{-1}\left[\left(x_{t}-\alpha_{t-1}\right)\left(x_{t-1}+\frac{x_{0}}{t+1}-\frac{t^{2}+3 t+1}{(t+1)^{2}} \alpha_{t-1}-\frac{1}{(t+1)^{2}} x_{t}\right)\right. \\
& \left.-\frac{t}{t+1} \beta_{t-1}\left(x_{t}-\alpha_{t-1}\right)^{2}\right], \\
R_{t} & =R_{t-1}+\frac{1}{t+1}\left[\frac{t}{t+1}\left(x_{t}-\alpha_{t-1}\right)^{2}-R_{t-1}\right] .
\end{aligned}\right.
$$


The actual law of motion under SAC-learning is therefore given by

$$
x_{t}=f\left(\alpha_{t-1}+\beta_{t-1}^{2}\left(x_{t-1}-\alpha_{t-1}\right), y_{t}, u_{t}\right),
$$

with $\alpha_{t}, \beta_{t}$ as in (2.8) and $y_{t}$ as in (2.2).

In Hommes and Sorger (1998), the map $f$ in (2.9) is a nonlinear deterministic function depending only on $\alpha_{t-1}+\beta_{t-1}^{2}\left(x_{t-1}-\alpha_{t-1}\right)$, without the driving variable $y_{t}$ and the noise $u_{t}$. Hommes et al. (2004) extend the CEE framework to SCEE, with $f$ a nonlinear stochastic process (but without exogenous driving variable $y_{t}$ ). In this paper the map $f$ is a linear function, depending on not only $\alpha_{t-1}+\beta_{t-1}^{2}\left(x_{t-1}-\alpha_{t-1}\right)$ and $u_{t}$ but also on an exogenous $\mathrm{AR}(1)$ process $y_{t}$. Hence, the true law of motion of the economy is a two dimensional linear stochastic process, while agents try to forecast using a univariate linear model. In the following we give two typical examples in economies and study existence of first-order SCEE and its relationship to SAC-learning in detail.

\section{An asset pricing model with $\mathrm{AR}(1)$ dividends}

A simple example of the general framework (2.1) is given by the standard present value asset pricing model with stochastic dividends; see for example Brock and Hommes (1998). Here we consider AR(1) dividends instead of independent and identically distributed (i.i.d.) dividends.

Assume that agents can invest in a risk free asset or in a risky asset. The risk-free asset is perfectly elastically supplied at a gross return $R>1$. $p_{t}$ denotes the price (ex dividend) of the risky asset and $y_{t}$ denotes the (random) dividend process. Let $\widetilde{E}_{t}, \widetilde{V}_{t}$ denote the subjective beliefs of a representative agent about the conditional expectation and conditional variance of excess return $p_{t+1}+y_{t+1}-R y_{t}$. By the assumption that the agent is a myopic mean-variance maximizer of tomorrow's wealth, the demand $z_{t}$ for the risky asset by the representative agent is then given by

$$
z_{t}=\frac{\widetilde{E}_{t}\left(p_{t+1}+y_{t+1}-R p_{t}\right)}{\widetilde{a} \widetilde{V}_{t}\left(p_{t+1}+y_{t+1}-R p_{t}\right)}=\frac{\widetilde{E}_{t}\left(p_{t+1}+y_{t+1}-R p_{t}\right)}{\widetilde{a} \sigma^{2}},
$$

where $\widetilde{a}>0$ denotes the risk aversion coefficient and the belief about the conditional variance of the excess return is assumed to be constant over time ${ }^{3}$, i.e. $\widetilde{V}_{t}\left(p_{t+1}+y_{t+1}-\right.$ $\left.R p_{t}\right) \equiv \sigma^{2}$

\footnotetext{
${ }^{3}$ This assumption is consistent with the assumption that agents believe that prices follow an AR(1) process and dividends follow a stochastic $\mathrm{AR}(1)$ process with finite variance.
} 
Equilibrium of demand and supply implies

$$
\frac{\widetilde{E}_{t}\left(p_{t+1}+y_{t+1}-R p_{t}\right)}{\widetilde{a} \sigma^{2}}=z_{s}
$$

where $z_{s}$ denotes the supply of outside shares in the market, assumed to be constant over time. Without loss of generality ${ }^{4}$, we assume zero supply of outside shares, i.e. $z_{s}=0$. The market clearing price in the standard asset pricing model is then given by

$$
p_{t}=\frac{1}{R}\left[p_{t+1}^{e}+y_{t+1}^{e}\right]
$$

where $p_{t+1}^{e}$ is the conditional expectation of next period's price $p_{t+1}$ and $y_{t+1}^{e}$ is the conditional expectation of next period's dividend $y_{t+1}$.

Dividend $\left\{y_{t}\right\}$ is assumed to follow an $\operatorname{AR}(1)$ process (2.2). Suppose that the risky asset (share) is traded, after payment of real dividends $y_{t}$, at a competitively determined price $p_{t}$, so that $y_{t}$ is known by agents, and

$$
y_{t+1}^{e}=a+\rho y_{t}
$$

The market clearing price in the standard asset pricing model with $\mathrm{AR}(1)$ dividends is then given by

$$
p_{t}=\frac{1}{R}\left[p_{t+1}^{e}+a+\rho y_{t}\right]
$$

where dividend $y_{t}$ follows the AR(1) process (2.2). Compared with our general framework (2.1), here the map $f$ is a simple linear function and the noise $u_{t} \equiv 0$.

\subsection{Rational expectations equilibrium with AR(1) dividends}

Under the assumption that agents are rational, a straightforward computation (see Appendix B) shows that the rational expectations equilibrium $p_{t}^{*}$ satisfies

$$
p_{t}^{*}=\frac{a R}{(R-1)(R-\rho)}+\frac{\rho}{R-\rho} y_{t} .
$$

In particular, if $\left\{y_{t}\right\}$ is i.i.d., i.e. $a=\bar{y}$ and $\rho=0$, then $p_{t}^{*}=\frac{a}{R-1}=\frac{\bar{y}}{R-1}$ for any $t=0,1,2, \cdots$.

Thus based on (3.4), the unconditional mean and the unconditional variance of the rational expectation price $p_{t}^{*}$ are given by, respectively,

$$
\begin{aligned}
\overline{p^{*}}:=E\left(p_{t}^{*}\right) & =\frac{a}{(R-1)(1-\rho)}=\frac{\bar{y}}{R-1}, \\
\operatorname{Var}\left(p_{t}^{*}\right) & =E\left(p_{t}^{*}-\overline{p^{*}}\right)^{2}=\frac{\rho^{2} \sigma_{\varepsilon}^{2}}{(R-\rho)^{2}\left(1-\rho^{2}\right)} .
\end{aligned}
$$

\footnotetext{
${ }^{4}$ In the case $z_{s}>0$, the difference with the analysis below only lies in the mean of the SCEE $\alpha^{*}=$ $\frac{\bar{y}-\widetilde{a} \sigma^{2} z_{s}}{R-1}$. The analysis on autocorrelations and variances remains the same.
} 
Furthermore, the first-order autocovariance and autocorrelation coefficient of the rational expectation price $p_{t}^{*}$ are given by, respectively,

$$
\begin{aligned}
E\left(p_{t}^{*}-\overline{p^{*}}\right)\left(p_{t-1}^{*}-\overline{p^{*}}\right) & =\frac{\rho^{3} \sigma_{\varepsilon}^{2}}{(R-\rho)^{2}\left(1-\rho^{2}\right)}, \\
\operatorname{Corr}\left(p_{t}^{*}, p_{t-1}^{*}\right) & =\rho .
\end{aligned}
$$

\subsection{Existence of first-order SCEE}

We now relax the rational expectation assumption and assume that agents are boundedly rational and believe that the price $p_{t}$ follows a univariate $\operatorname{AR}(1)$ process

$$
p_{t}=\alpha+\beta\left(p_{t-1}-\alpha\right)+\delta_{t}
$$

Given the perceived law of motion and knowledge of all prices observed up to period $t-1$, the 2-period ahead forecasting rule for $p_{t+1}$ that minimizes the mean-squared forecasting error is

$$
p_{t+1}^{e}=\alpha+\beta^{2}\left(p_{t-1}-\alpha\right)
$$

By substituting (3.9) into (3.3), we obtain the implied actual law of motion for prices

$$
\left\{\begin{array}{l}
p_{t}=\frac{1}{R}\left[\alpha+\beta^{2}\left(p_{t-1}-\alpha\right)+a+\rho y_{t}\right], \\
y_{t}=a+\rho y_{t-1}+\varepsilon_{t} .
\end{array}\right.
$$

For the PLM (3.8) and the ALM (3.10), we first study the existence and uniqueness of first-order SCEE.

Since $0 \leq \frac{\beta^{2}}{R}<1$ and $0 \leq \rho<1$, the price process (3.10) is stationary and ergodic. Denote the unconditional expectation of $p_{t}$ by $\bar{p}$. Then $\bar{p}$ satisfies

$$
R \bar{p}=\alpha\left(1-\beta^{2}\right)+\beta^{2} \bar{p}+a+\rho \bar{y}=\alpha\left(1-\beta^{2}\right)+\beta^{2} \bar{p}+\bar{y}
$$

Hence

$$
\bar{p}=\frac{\alpha\left(1-\beta^{2}\right)+\bar{y}}{R-\beta^{2}} .
$$

Imposing the first consistency requirement of a SCEE on the mean, i.e. $\bar{p}=\alpha$, yields

$$
\alpha=\frac{\bar{y}}{R-1}=: \alpha^{*}
$$

Hence using (3.5), we conclude that in a SCEE the unconditional mean of market prices coincides with the REE fundamental prices. That is to say, in a SCEE market prices fluctuate around the fundamental prices. 
Next consider the second consistency requirement of a SCEE on the first-order autocorrelation coefficient $\beta$ of the PLM. A straightforward computation (see Appendix C) shows that the first-order autocorrelation coefficient $\operatorname{Corr}\left(p_{t}, p_{t-1}\right)$ of the ALM satisfies

$$
\operatorname{Corr}\left(p_{t}, p_{t-1}\right)=\frac{\beta^{2}+R \rho}{\rho \beta^{2}+R}=: F(\beta) \text {. }
$$

Define $G(\beta):=F(\beta)-\beta$. In the case that $\rho>0^{5}$, since $G(0)=\rho>0$ and $G(1)=$ $\frac{1+R \rho}{\rho+R}-1=\frac{(1-\rho)(1-R)}{\rho+R}=\frac{-r(1-\rho)}{\rho+R}<0$, there exists at least one $\beta^{*} \in(0,1)$, such that $G\left(\beta^{*}\right)=0$, i.e.

$$
F\left(\beta^{*}\right)=\beta^{*}
$$

Furthermore, because $F(0)=\rho$ and $F^{\prime}(\beta)=\frac{2 \beta R\left(1-\rho^{2}\right)}{\left(\rho \beta^{2}+R\right)^{2}}>0$ for $\beta \in(0,1)$, we have $F(\beta)>\rho$ for $\beta \in(0,1)$. Hence

$$
\beta^{*}>\rho
$$

It can be shown (see Appendix D) that $\beta^{*}$ is unique. We thus have the following proposition on first-order stochastic consistent expectations equilibrium.

Proposition 1 In the case that $0<\rho<1$, there exists a unique nonzero first-order stochastic consistent expectations equilibrium $\left(\alpha^{*}, \beta^{*}\right)$ for the asset pricing model with $A R(1)$ dividends (3.10), which satisfies $\alpha^{*}=\frac{\bar{y}}{(R-1)}=\overline{p^{*}}$ and $\beta^{*}>\rho$.

This proposition states that in a SCEE self-fulfilling market prices have the same mean as the fundamental prices, but a higher first-order autocorrelation coefficient than the fundamental prices. In other words, in a SCEE market prices fluctuate around the fundamental prices but with a higher persistence than under REE.

\subsubsection{Numerical analysis}

Now we illustrate the above results numerically. For example, consider $R=1.05, \rho=$ $0.9, a=0.005, \varepsilon_{t} \sim$ i.i.d. $U(-0.01,0.01 \text { ) (i.e. uniform distribution on }[-0.01,0.01])^{6}$. Figures $1 \mathrm{a}^{7}$ and $1 \mathrm{~b}$ illustrate the existence of a unique stable first-order SCEE, where $\left(\alpha^{*}, \beta^{*}\right)=(1,0.997)$. The time series of fundamental prices and market prices with

\footnotetext{
${ }^{5}$ In the case that $\rho=0, F(\beta)=\frac{\beta^{2}}{R}$, which can be obtained from (3.13). Hence $G(\beta)=\frac{\beta^{2}}{R}-\beta=\frac{\beta(\beta-R)}{R}$.
} Since $\beta \leq 1<R$, the only equilibrium is $\beta=0$. Therefore, in the case that $\rho=0$, there is no nonzero first-order stochastic consistent expectations equilibrium (SCEE).

${ }^{6}$ As shown theoretically above, the numerical results are independent of selection of the parameter values within plausible ranges, sample paths, initial values and distribution of noise.

${ }^{7}$ In Figure 1a, we take $\beta=0.9$. However in fact, $\alpha^{*}$ is independent of $\beta$, as can be seen from (3.12). 
$(\alpha, \beta)=\left(\alpha^{*}, \beta^{*}\right)$ are shown in Figure 1c, which illustrates that the market price fluctuates around the fundamental price but has more persistence and exhibits excess volatility. In fact, based on Proposition 1, in a SCEE the mean of the market prices is equal to that of the fundamental prices and the first-order autocorrelation coefficient $\beta^{*}$ of the market prices is greater than that of the fundamental prices $\rho$, implying that the market prices have higher persistence. In order to further illustrate this, the autocorrelation functions of the market prices and the fundamental prices are shown in Figure 1d. It can be seen from Figure 1d that autocorrelation coefficients of the market prices are higher than those of the fundamental prices and hence the market prices have higher persistence.

We now investigate how the first-order SCEE and excess volatility of market prices depend on the autoregressive coefficient of dividends $\rho$, which is also the first-order autocorrelation of fundamental prices. Consistent with Proposition 1, Figure 2a illustrates that the first-order autocorrelation of market prices is higher than that of fundamental prices, especially much higher as $\rho>0.4$. For $\rho \geq 0.5$ we have $\beta^{*}>0.9$, implying that in equilibrium asset prices are close to a random walk and therefore quite unpredictable. In fact, based on empirical findings, e.g. Timmermann (1996), the autoregressive coefficient of dividends $\rho$ is about 0.9 , where the corresponding $\beta^{*} \approx 0.997$, very close to a random walk. In the case $\rho>0.4$, correspondingly the variance of market prices is larger than that of fundamental prices, as illustrated in Figure 2b. In the Figure 2b, the ratio of variance of market prices and variance of fundamental prices is greater than 1 for $0.4<\rho<1$. For $\rho=0.9, \frac{\sigma_{p}^{2}}{\sigma_{p^{*}}^{2}} \approx 2.5$. Given the variance of fundamental prices (3.6) and the variance of market prices (C.6),

$$
\frac{\sigma_{p}^{2}}{\sigma_{p^{*}}^{2}}=\left.\frac{\left(\beta^{2} \rho+R\right)(R-\rho)^{2}}{\left(R^{2}-\beta^{4}\right)\left(R-\rho \beta^{2}\right)}\right|_{\beta=\beta^{*}(\rho)} .
$$

Proposition 1 demonstrates $\rho<\beta^{*}(\rho)<1$ for $0<\rho<1$, and hence $\beta^{*}(\rho)$ converges to 1 as $\rho$ tends to 1 . Thus as $\rho$ tends to $1, \frac{\sigma_{p}^{2}}{\sigma_{p^{*}}^{2}}$ converges to 1 , consistent with Figure $2 \mathrm{~b}$. So for plausible parameter values of $\rho$, the variance of market prices is greater than that of fundamental prices, indicating that market prices have excess volatility in the SCEE.

\subsection{Stability under SAC-learning}

In this subsection we study the stability of SCEE under SAC-learning in the asset pricing model with $\mathrm{AR}(1)$ dividends. The asset pricing model with $\mathrm{AR}(1)$ dividends 


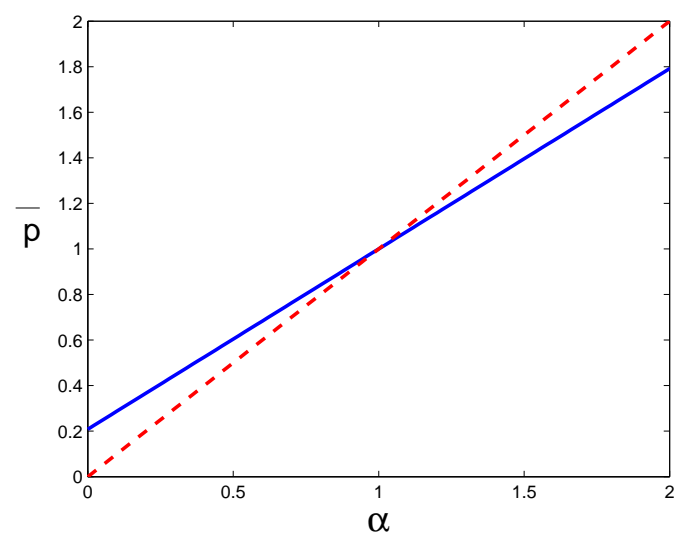

(a) SCEE for $\alpha^{*}$

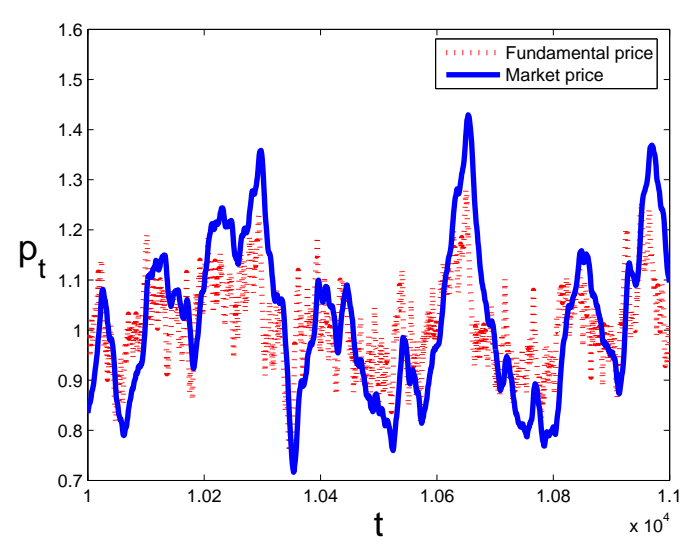

(c) Time series of prices in SCEE

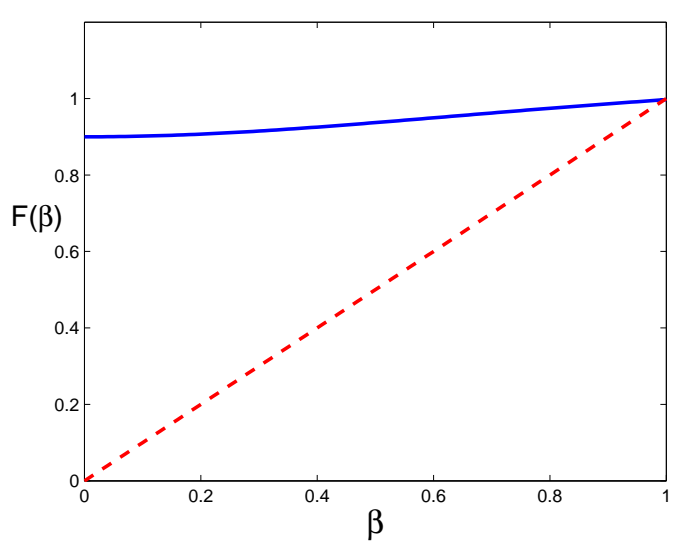

(b) SCEE for $\beta^{*}$

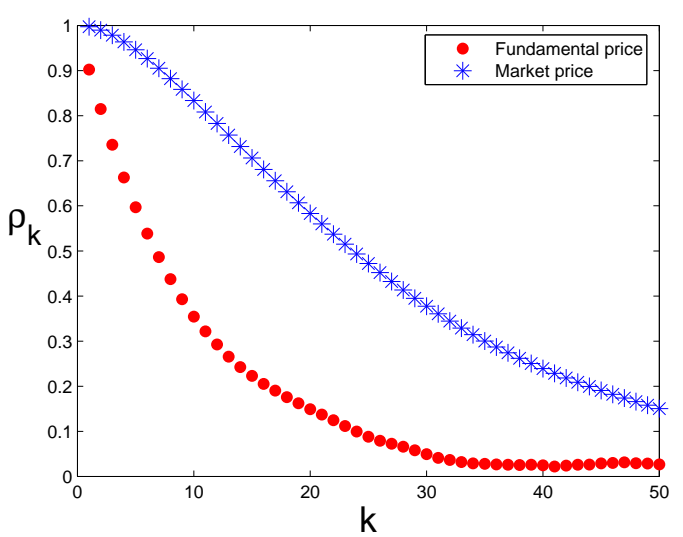

(d) Autocorrelation function in SCEE

Figure 1: (a) SCEE $\alpha^{*}(=1)$ is the intersection point of the mean $\bar{p}=\frac{\alpha\left(1-\beta^{2}\right)+\bar{y}}{R-\beta^{2}}$ (bold curve) with the perceived mean $\alpha$ (dotted line); (b) $\operatorname{SCEE~} \beta^{*}(=0.997)$ is the intersection point of the first-order autocorrelation coefficient $F(\beta)=\frac{\beta^{2}+R \rho}{\rho \beta^{2}+R}$ (bold curve) with the perceived first-order autocorrelation $\beta$ (dotted line); (c) 1,000 observations of fundamental prices (dotted curve) and market prices (bold curve) in the SCEE; (d) autocorrelation functions of 10,000 fundamental prices (lower dots) and market prices (higher stars) in the SCEE. Parameter values are $R=1.05, \rho=0.9, a=0.005, \varepsilon_{t} \sim$ i.i.d. $U(-0.01,0.01)$. 


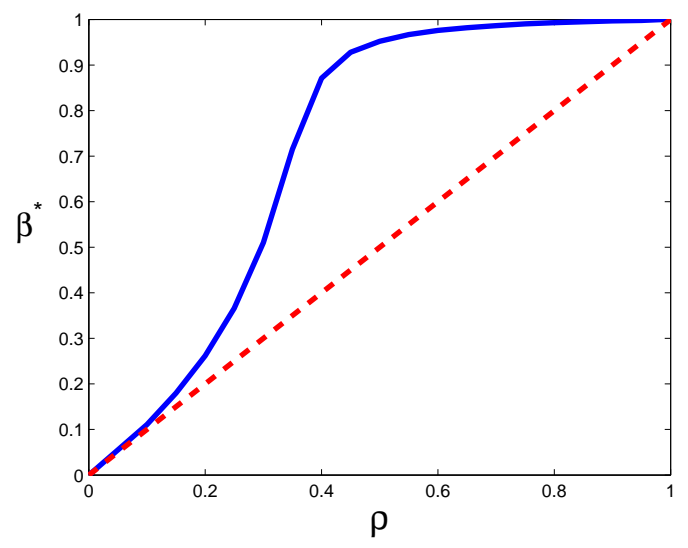

(a) 1-order autocorrelation in SCEE

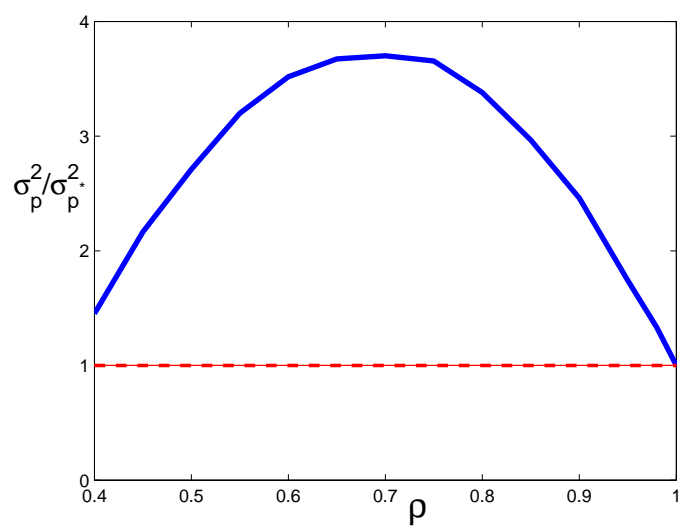

(b) Ratio of variances in SCEE

Figure 2: (a) SCEE $\beta^{*}$ with respect to $\rho$; (b) ratio of variance of market prices and variance of fundamental prices with respect to $\rho$, where $R=1.05$.

under SAC-learning is given by

$$
\left\{\begin{array}{l}
p_{t}=\frac{1}{R}\left[\alpha_{t-1}+\beta_{t-1}^{2}\left(p_{t-1}-\alpha_{t-1}\right)+a+\rho y_{t}\right], \\
y_{t}=a+\rho y_{t-1}+\varepsilon_{t},
\end{array}\right.
$$

with $\alpha_{t}, \beta_{t}$ as in (2.8). This is an expectations feedback system. Realized prices influence the perceptions agents have about economic reality and these perceptions feed back into the actual dynamics of the economy and determine future prices that will be realized.

In order to study the dynamical behavior of the model (3.14), we first check the stability of the unique SCEE $\left(\alpha^{*}, \beta^{*}\right)$ in Proposition 1 . The stability of the SCEE is in fact determined by the coefficient $\frac{1-\beta^{2}}{R-\beta^{2}}$ in front of $\alpha$ in the unconditional mean in (3.11) and by $F(\beta)$ in (3.13). On one hand, since $0 \leq \frac{1-\beta^{2}}{R-\beta^{2}}<1$, it can be seen from (3.11) that $\alpha^{*}$ is stable. On the other hand, the proof of uniqueness of $\beta^{*}$ in Appendix $\mathrm{D}$ shows that $0<F^{\prime}\left(\beta^{*}\right)<1$, and that therefore $\beta^{*}$ is stable. We thus have stability of the unique SCEE under SAC-learning.

Proposition 2 The unique SCEE $\left(\alpha^{*}, \beta^{*}\right)$ in Proposition 1 is stable under SAC-learning, that is, the SAC-learning process $\left(\alpha_{t}, \beta_{t}\right)$ converges to the unique SCEE $\left(\alpha^{*}, \beta^{*}\right)$ as time $t$ tends to $\infty$.

Proof. See Appendix E.

This proposition shows that the SCEE describes the long-run behavior of SAC-learning when agents use a simple $\mathrm{AR}(1)$ forecasting rule. 


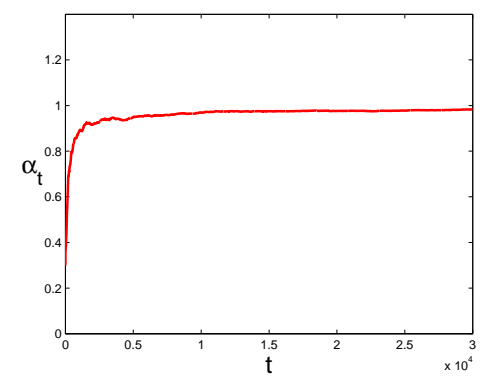

(a)

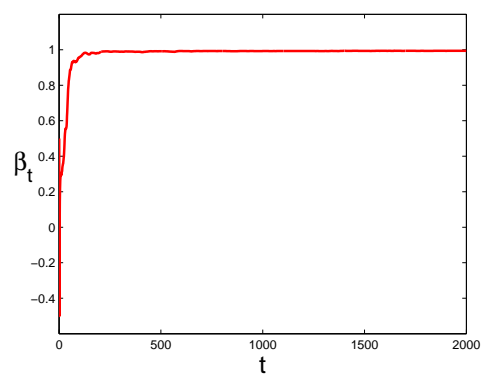

(b)

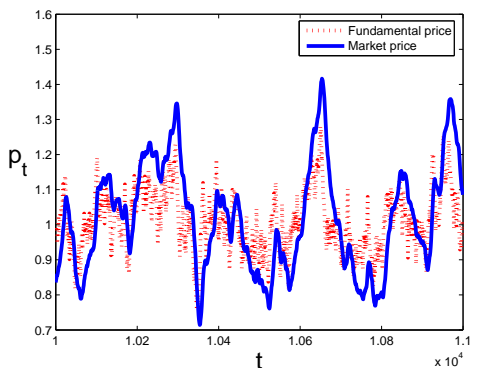

(c)

Figure 3: (a) Time series $\alpha_{t} \rightarrow \alpha^{*}(1.0)$ under SAC-learning; (b) time series $\beta_{t} \rightarrow \beta^{*}(0.997)$ under SAC-learning; (c) time series of market prices under SAC-learning and fundamental prices. Initial values $p_{0}=0.3, y_{0}=0.08$.

\subsubsection{Numerical analysis}

Figure 3 shows that SAC-learning $\left(\alpha_{t}, \beta_{t}\right)$ converges to the unique stable $\operatorname{SCEE~}\left(\alpha^{*}, \beta^{*}\right)$. Figure $3 \mathrm{a}$ indicates that the mean of the market prices under SAC-learning $\alpha_{t}$ tends to the mean $\alpha^{*}=1$ in the SCEE, while Figure $3 \mathrm{~b}$ shows that the first-order autocorrelation coefficient of the market prices under SAC-learning $\beta_{t}$ tends to the first-order autocorrelation coefficient $\beta^{*}=0.997$ in the SCEE. Therefore, given the same sample path of noise, the time series of the market prices under SAC-learning is almost the same as that in the SCEE, which can be seen by comparing Figure 3c to Figure 1c. That is, the market prices under SAC-learning fluctuate around the fundamental prices but have excess volatility and stronger autocorrelation. Therefore, the self-referential SCEE and learning offer a possible explanation of bubbles within a stationary time series framework, as suggested in Bullard et al. (2010).

\section{The New Keynesian Philips curve with AR(1) driv- ing variable}

Now consider a second application of SCEE and learning in macroeconomics, the New Keynesian Philips curve with an AR(1) driving variable as suggested by Lansing (2009). Assume that the inflation and the output gap (real marginal cost) evolve according to

$$
\left\{\begin{array}{l}
\pi_{t}=\lambda \pi_{t+1}^{e}+\gamma y_{t}+u_{t}, \\
y_{t}=a+\rho y_{t-1}+\varepsilon_{t},
\end{array}\right.
$$


where $\pi_{t}$ is the inflation at time $\mathrm{t}, \pi_{t+1}^{e}$ is expected inflation at date $t+1$ and $y_{t}$ is the output gap or real marginal cost. $\lambda \in[0,1)$ is the representative agent's subjective time discount factor, $\gamma>0$ is related to the degree of price stickiness in the economy and $\rho \in[0,1)$ describes the linear dependence of the output gap on its past value. $u_{t}$ and $\varepsilon_{t}$ are i.i.d. stochastic disturbances with zero mean and finite absolute moments with variances $\sigma_{u}^{2}$ and $\sigma_{\varepsilon}^{2}$, respectively. The key difference with the standard asset pricing model is that this model includes two stochastic disturbances, not only the noise $\varepsilon_{t}$ of the $\mathrm{AR}(1)$ driving variable, but also an additional noise $u_{t}$ in the New Keynesian Philips curve. We refer to $u_{t}$ as a markup shock that is often motivated by the presence of a variable tax rate and to $\varepsilon_{t}$ as a demand shock that is uncorrelated with the markup shock.

\subsection{Rational expectations equilibrium}

If agents are rational, then a straightforward computation (see Appendix F) gives the rational expectations equilibrium

$$
\pi_{t}^{*}=\frac{\gamma \lambda a}{(1-\lambda)(1-\lambda \rho)}+\frac{\gamma}{1-\lambda \rho} y_{t}+u_{t}
$$

Hence the mean and variance of rational expectations equilibrium $\pi_{t}^{*}$ are, respectively,

$$
\begin{aligned}
\overline{\pi^{*}}:=E\left(\pi_{t}^{*}\right) & =\frac{\gamma a}{(1-\lambda)(1-\rho)} \\
\operatorname{Var}\left(\pi_{t}^{*}\right) & =E\left(\pi_{t}^{*}-\overline{\pi^{*}}\right)^{2}=\frac{\gamma^{2} \sigma_{\varepsilon}^{2}}{(1-\lambda \rho)^{2}\left(1-\rho^{2}\right)}+\sigma_{u}^{2} .
\end{aligned}
$$

Furthermore, the first-order autocovariance and autocorrelation of rational expectations equilibrium $\pi_{t}^{*}$ are, respectively,

$$
\begin{aligned}
E\left(\pi_{t}^{*}-\overline{\pi^{*}}\right)\left(\pi_{t-1}^{*}-\overline{\pi^{*}}\right) & =\frac{\gamma^{2} \rho \sigma_{\varepsilon}^{2}}{(1-\lambda \rho)^{2}\left(1-\rho^{2}\right)} \\
\operatorname{Corr}\left(\pi_{t}^{*}, \pi_{t-1}^{*}\right) & =\frac{\rho \gamma^{2}}{\gamma^{2}+(1-\lambda \rho)^{2}\left(1-\rho^{2}\right) \frac{\sigma_{u}^{2}}{\sigma_{\varepsilon}^{2}}} .
\end{aligned}
$$

Note that in the special case $\sigma_{u}^{2}=0$, the above expression reduces to $\operatorname{Corr}\left(\pi_{t}^{*}, \pi_{t-1}^{*}\right)=\rho$ as in Eq. (3.7). Moreover, the larger the noise level $\sigma_{u}^{2}$ in the markup shock, the smaller the first-order autocorrelation in the fundamental rational equilibrium inflation. 


\subsection{Existence of first-order SCEE}

Suppose now that agents are boundedly rational and that their perceived law of motion for inflation is a univariate $\operatorname{AR}(1)$ process.

$$
\pi_{t}=\alpha+\beta\left(\pi_{t-1}-\alpha\right)+v_{t}
$$

The implied actual law of motion then becomes

$$
\left\{\begin{array}{l}
\pi_{t}=\lambda\left[\alpha+\beta^{2}\left(\pi_{t-1}-\alpha\right)\right]+\gamma y_{t}+u_{t} \\
y_{t}=a+\rho y_{t-1}+\varepsilon_{t}
\end{array}\right.
$$

Denote the unconditional expectation of $\pi_{t}$ by $\bar{\pi}$ and the unconditional expectation of $y_{t}$ by $\bar{y}$. Then $\bar{y}=a /(1-\rho)$ and $\bar{\pi}$ satisfies

$$
\bar{\pi}=\lambda \alpha\left(1-\beta^{2}\right)+\lambda \beta^{2} \bar{\pi}+\gamma \bar{y}
$$

Hence

$$
\bar{\pi}=\frac{\lambda \alpha\left(1-\beta^{2}\right)+\gamma \bar{y}}{1-\lambda \beta^{2}}
$$

Imposing the first consistency requirement on the mean, i.e. $\bar{\pi}=\frac{\lambda \alpha\left(1-\beta^{2}\right)+\gamma \bar{y}}{1-\lambda \beta^{2}}=\alpha$, we get

$$
\alpha=\frac{\gamma \bar{y}}{1-\lambda}=\frac{\gamma a}{(1-\lambda)(1-\rho)}=: \alpha^{*}
$$

Therefore using (4.3), in a SCEE the unconditional mean of inflation coincides with the REE fundamental inflation.

After straightforward computations (see Appendix G), we obtain

$$
\operatorname{Corr}\left(\pi_{t}, \pi_{t-1}\right)=\frac{\gamma^{2}\left(\lambda \beta^{2}+\rho\right)+\lambda \beta^{2}\left(1-\rho^{2}\right)\left(1-\lambda \beta^{2} \rho\right) \frac{\sigma_{u}^{2}}{\sigma_{\varepsilon}^{2}}}{\gamma^{2}\left(\lambda \beta^{2} \rho+1\right)+\left(1-\rho^{2}\right)\left(1-\lambda \beta^{2} \rho\right) \frac{\sigma_{u}^{2}}{\sigma_{\varepsilon}^{2}}}=: \bar{F}(\beta)
$$

Note that if we replace $\lambda$ by $\frac{1}{R}, \gamma$ by $\frac{\rho}{R}$ and $\sigma_{u}$ by 0 , then the autocorrelation in (4.8) is simplified to $\frac{\beta^{2}+R \rho}{\rho \beta^{2}+R}$, which coincides with the autocorrelation in the asset pricing model in $(3.13)$.

The second consistency requirement of first-order autocorrelation coefficient $\beta$ yields,

$$
\bar{F}(\beta)=\beta .
$$

Define $\bar{G}(\beta):=\bar{F}(\beta)-\beta$. Since $0<\rho<1$ and $0 \leq \lambda<1$,

$$
\bar{G}(0)=\frac{\gamma^{2} \rho}{\gamma^{2}+\left(1-\rho^{2}\right) \frac{\sigma_{u}^{2}}{\sigma_{\varepsilon}^{2}}}>0
$$


and

$$
\begin{aligned}
\bar{G}(1) & =\frac{\gamma^{2}(\lambda+\rho)+\lambda\left(1-\rho^{2}\right)(1-\lambda \rho) \frac{\sigma_{u}^{2}}{\sigma_{\varepsilon}^{2}}}{\gamma^{2}(\lambda \rho+1)+\left(1-\rho^{2}\right)(1-\lambda \rho) \frac{\sigma_{u}^{2}}{\sigma_{\varepsilon}^{2}}}-1 \\
& =\frac{-\gamma^{2}(1-\lambda)(1-\rho)-(1-\lambda)\left(1-\rho^{2}\right)(1-\lambda \rho) \frac{\sigma_{u}^{2}}{\sigma_{\varepsilon}^{2}}}{\gamma^{2}(\lambda \rho+1)+\left(1-\rho^{2}\right)(1-\lambda \rho) \frac{\sigma_{u}^{2}}{\sigma_{\varepsilon}^{2}}} \\
& <0 .
\end{aligned}
$$

Therefore, there exists at least one $\beta^{*} \in(0,1)$, such that $\bar{G}\left(\beta^{*}\right)=0$, i.e. $\bar{F}\left(\beta^{*}\right)=\beta^{*}$. In the special case without autocorrelation in the driving variable $y_{t}$, i.e. $\rho=0$, equation (4.8) gives $\bar{F}(\beta)=\lambda \beta^{2}$. Hence the first-order SCEE for $\rho=0$ is $\beta^{*}=0$ and coincides with the REE.

Proposition 3 In the case that $0<\rho<1$ and $0 \leq \lambda<1$, there exists at least one nonzero first-order stochastic consistent expectations equilibrium (SCEE) $\left(\alpha^{*}, \beta^{*}\right)$ for the New Keynesian Philips curve (4.6) with $\alpha^{*}=\frac{\gamma a}{(1-\lambda)(1-\rho)}=\overline{\pi^{*}}$.

It turns out that in the NKPC multiple SCEE may co-exist. To see this, rewrite the first-order autocorrelation, $\bar{F}(\beta)=\lambda \beta^{2}+\frac{\rho\left(1-\lambda^{2} \beta^{4}\right)}{\left(\lambda \beta^{2} \rho+1\right)+\left(1-\rho^{2}\right)\left(1-\lambda \beta^{2} \rho\right) \frac{1}{\gamma^{2}} \cdot \frac{\sigma_{u}^{2}}{\sigma_{\varepsilon}^{2}}}$. It is easy to see that if $\gamma$ or $\frac{\sigma_{\varepsilon}^{2}}{\sigma_{u}^{2}}$ increases, then $\bar{F}(\beta)$ increases, and therefore multiple SCEE may occur. The simulations in the following subsection illustrate this point more clearly.

\subsubsection{Numerical analysis}

Now we illustrate the existence of SCEE and the effects of $\rho, \gamma$ and $\frac{\sigma_{\varepsilon}^{2}}{\sigma_{u}^{2}}$ numerically. Based on empirical findings, such as Lansing (2009), Gali et al. (2001) and Fuhrer (2006, 2009), we first examine a plausible case ${ }^{8}$ in which $\gamma=0.075, \sigma_{u}=0.003162, \sigma_{\varepsilon}=0.01, \rho=$ $0.9, \lambda=0.99, \varepsilon_{t} \sim N\left(0, \sigma_{\varepsilon}^{2}\right), u_{t} \sim N\left(0, \sigma_{u}^{2}\right), a=0.0004$. Hence $\frac{\sigma_{u}^{2}}{\sigma_{\varepsilon}^{2}}=0.1$. Figure 4a illustrates existence of a unique (stable) $\alpha^{*}$, where $\alpha^{*}=0.03$. Figure $4 \mathrm{~b}$ shows that there exist three $\beta^{*}$, where $\beta^{*}=0.3066,0.7417,0.9961$. That is, there exist three firstorder SCEE: two stable ones $\left(\alpha^{*}, \beta^{*}\right)=(0.03,0.3066),(0.03,0.9961)$ and an unstable

\footnotetext{
${ }^{8}$ As shown in Lansing (2009), based on regressions using either the output gap or labor's share of income over the period 1949.Q1 to 2004.Q4, $\rho=0.9, \sigma_{\varepsilon}=0.01$. Estimates of the NKPC parameters $\lambda, \gamma, \sigma_{u}$ are sensitive to the choice of the driving variable, the sample period, and the econometric model etc. Later we also examine the effects of some other parameters on SCEE. Furthermore, based on the above theoretical results, $a$ just affects the mean of inflation $\bar{\pi}$ but not the autocorrelation coefficient $\bar{F}(\beta)$. For $\sigma_{u}$ and $\sigma_{\varepsilon}, \bar{F}(\beta)$ only depends on their ratio $\sigma_{u} / \sigma_{\varepsilon}$ but not on their absolute values.
} 
one $\left(\alpha^{*}, \beta^{*}\right)=(0.03,0.7417)$. Considering that the SAC-learning converges to a stable SCEE (see the next subsection), the stable (learnable) SCEE are the most interesting. Figures $4 \mathrm{c}$ and $4 \mathrm{~d}$ illustrate the two time series for the two (stable) $\operatorname{SCEE}\left(\alpha^{*}, \beta^{*}\right)=$ $(0.03,0.3066),(0.03,0.9961)$, suggesting that inflation has different persistence at different SCEE. That is, the SCEE is an important factor in affecting inflation persistence. In fact, the time series of inflation in the SCEE with high $\beta^{*}$ in Figure $4 \mathrm{~d}$ has similar persistence characteristics and amplitude of fluctuation as in empirical inflation data in Tallman (2003). Furthermore, Figure 4d illustrates that inflation in the SCEE with high $\beta^{*}$ has stronger persistence than the REE inflation, where the first-order autocorrelation coefficient of REE inflation is 0.865 less than $\beta^{*}=0.9961$.

In order to further study the effects of $\rho$, Figure 5 illustrates SCEE $\beta^{*}$ together with the first-order autocorrelation coefficient of REE inflation as functions of $\rho$. For $0.84<\rho<0.918$, two stable SCEE $\beta^{*}$ exist separated by an unstable SCEE. The large $\operatorname{SCEE~} \beta^{*}$ is larger than the first-order autocorrelation coefficient of REE inflation, while the small SCEE $\beta^{*}$ is smaller than the first-order autocorrelation coefficient of REE inflation. In the next subsection we will show that for a large range of initial values of inflation the SAC-learning converges to the stable high SCEE $\beta^{*}$ with strong persistence. If $\rho>0.918$, there exists only one stable $\operatorname{SCEE~} \beta^{*}$ with stronger persistence than REE. Therefore for plausible values of $\rho$ around 0.9 , inflation in a SCEE often generates highpersistence as shown in Figure 4d. This result is consistent with the empirical finding in Adam (2007) that the Restricted Receptions Equilibrium (RPE) describes subjects' inflation expectations surprisingly well and provides a better explanation for the observed persistence of inflation than REE.

Figure 6 illustrates how the number of SCEE depends on $\gamma$. The simulations show that for plausible $\gamma$ there exist at least one and at most three SCEE $\beta^{*}$. For sufficiently small $\gamma(<0.05)$, there exists only one low $\beta^{*}$, as shown in Figure 6a. As $\gamma$ increases, the graph of $\bar{F}(\beta)=\lambda \beta^{2}+\frac{\rho\left(1-\lambda^{2} \beta^{4}\right)}{\left(\lambda \beta^{2} \rho+1\right)+\left(1-\rho^{2}\right)\left(1-\lambda \beta^{2} \rho\right) \frac{1}{\gamma^{2}} \cdot \frac{\sigma_{u}^{2}}{\sigma_{\varepsilon}^{2}}}$ goes up. At $\gamma=0.05$, a new SCEE $\beta^{*} \approx 0.975$ is created at a tangent bifurcation, see Figure $6 \mathrm{~b}$. Immediately after that there exist three $\beta^{*}$, two stable equilibria and one unstable. That is, at $\gamma=0.05$, a tangent bifurcation occurs. Figures $6 \mathrm{c}$ and $6 \mathrm{~d}$ illustrate the three $\beta^{*}$ with the high stable $\beta^{*}$ close to 1 . As $\gamma$ increases, the two stable $\beta^{*}$-values grow. At $\gamma=0.084$, another tangent bifurcation occurs, where the lower $\beta^{*}$-values coincide, as shown in Figure 6e. For $\gamma>0.084$, there is only one large $\beta^{*}$, see Figure 6 f, which corresponds to high-persistence SCEE of inflation. 


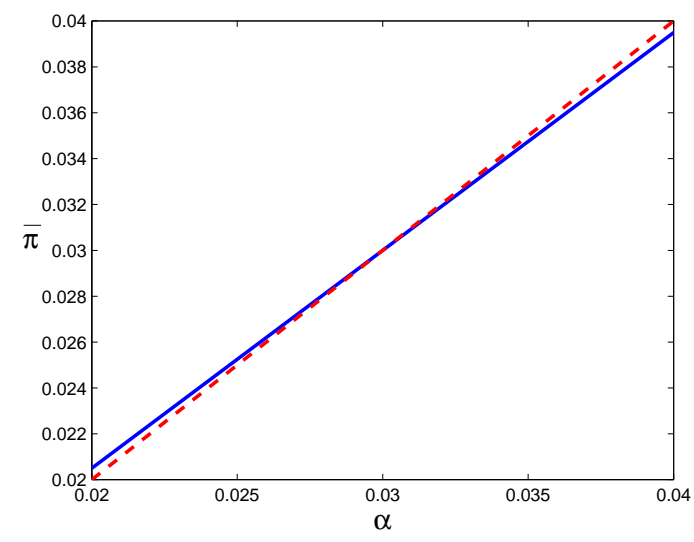

(a) SCEE for $\alpha^{*}$

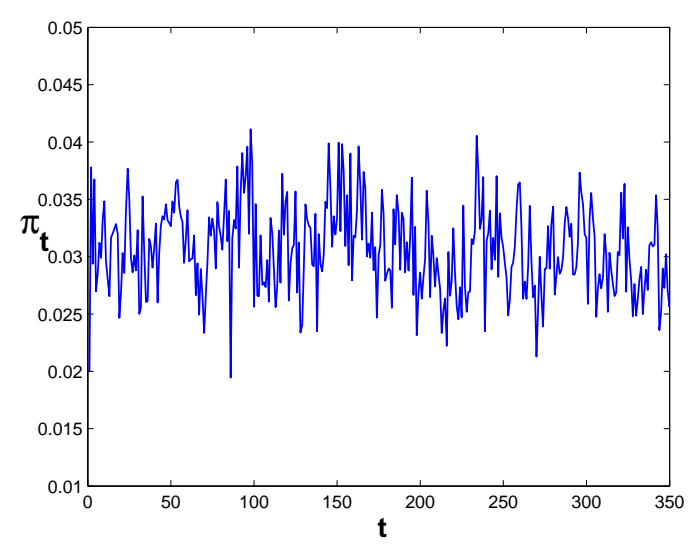

(c) time series at $\left(\alpha^{*}, \beta^{*}\right)=(0.03,0.3066)$

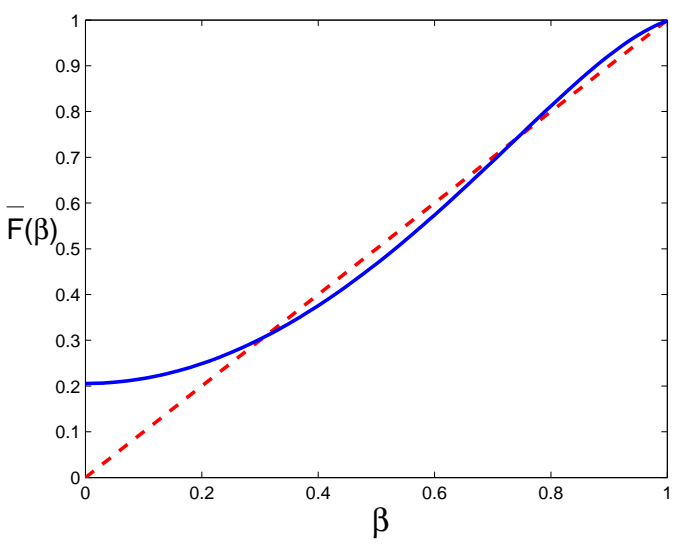

(b) SCEE for $\beta^{*}$

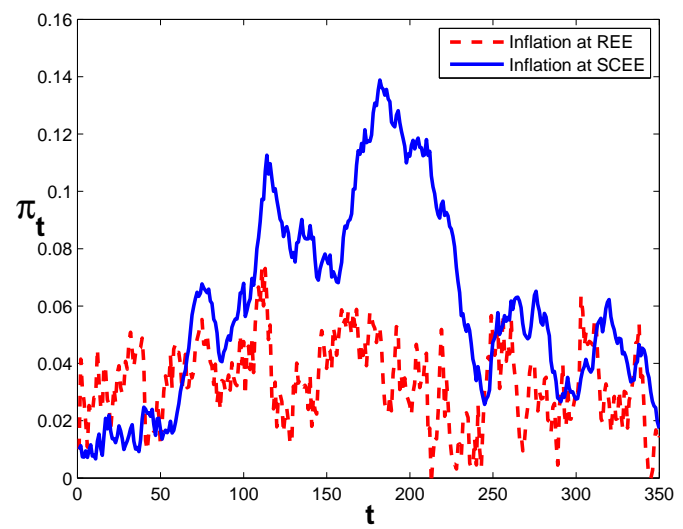

(d) time series at $\left(\alpha^{*}, \beta^{*}\right)=(0.03,0.9961)$

Figure 4: (a) SCEE $\alpha^{*}$ is the unique intersection point of the mean of inflation $\bar{\pi}=$ $\frac{\lambda \alpha\left(1-\beta^{2}\right)+\gamma \bar{y}}{1-\lambda \beta^{2}}$ (bold curve) with the perceived mean $\alpha$ (dotted line); (b) SCEE $\beta^{*}$ is an intersection point of the first-order autocorrelation of inflation $\frac{\gamma^{2}\left(\lambda \beta^{2}+\rho\right)+\lambda \beta^{2}\left(1-\rho^{2}\right)\left(1-\lambda \beta^{2} \rho\right) \frac{\sigma_{u}^{2}}{\sigma_{\varepsilon}^{2}}}{\gamma^{2}\left(\lambda \beta^{2} \rho+1\right)+\left(1-\rho^{2}\right)\left(1-\lambda \beta^{2} \rho\right) \frac{\sigma_{u}^{2}}{\sigma_{\varepsilon}^{2}}}$ (bold curve) and the perceived first-order autocorrelation $\beta$ (dotted line); (c) time series of inflation in stable low-persistence $\operatorname{SCEE}\left(\alpha^{*}, \beta^{*}\right)=(0.03,0.3066)$; (d) times series of inflation in stable high-persistence $\operatorname{SCEE}\left(\alpha^{*}, \beta^{*}\right)=(0.03,0.9961)$ (bold curve) and time series of REE inflation (dotted curve), where $\gamma=0.075, \sigma_{u}=0.003162, \sigma_{\varepsilon}=0.01, \rho=$ $0.9, \lambda=0.99, \varepsilon_{t} \sim N\left(0, \sigma_{\varepsilon}^{2}\right), u_{t} \sim N\left(0, \sigma_{u}^{2}\right), a=0.0004$ 


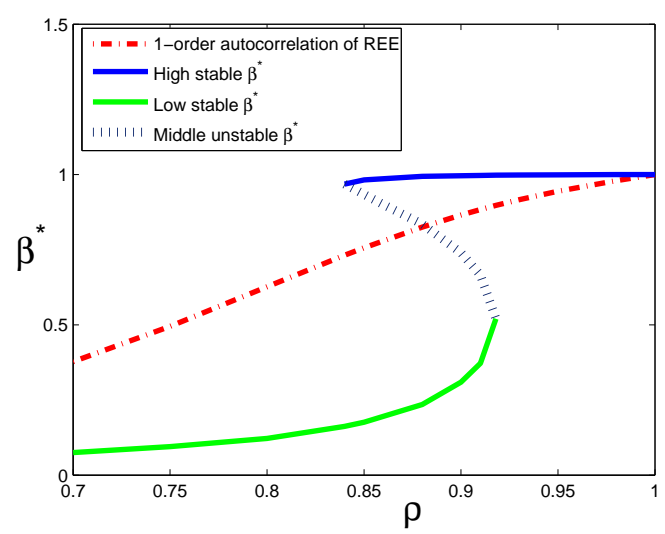

Figure 5: First-order autocorrelation coefficient of REE inflation (dotted real curve), stable SCEE $\beta^{*}$ with respect to $\rho$ (bold curves), unstable SCEE $\beta^{*}$ (dotted curve), where $\gamma=0.075, \sigma_{u}=0.003162, \sigma_{\varepsilon}=0.01, \lambda=0.99$.

Hence a larger $\gamma$ tends to lead to higher persistence of inflation. Intuitively with a larger $\gamma$, it can be seen from (4.1) that the driving variable (output gap or real marginal cost) has a larger impact on inflation. Hence when the driving variable is relatively important, a high-persistence SCEE occurs. If on the other hand, $\sigma_{u}^{2}$ increases, that is, the noise to inflation increases, the ratio $\frac{\sigma_{\varepsilon}^{2}}{\sigma_{u}^{2}}$ decreases and the strong reverses and low-persistence SCEE become more likely. That is, intuitively clear, as more noise to inflation dominates the driving variable, this leads to a low-persistence inflation equilibrium.

\subsection{Stability under SAC-learning}

The SAC-learning dynamics in the New Keynesian Philips curve with AR(1) driving variable is given by

$$
\left\{\begin{array}{l}
\pi_{t}=\lambda\left[\alpha_{t-1}+\beta_{t-1}^{2}\left(\pi_{t-1}-\alpha_{t-1}\right)\right]+\gamma y_{t}+u_{t} \\
y_{t}=a+\rho y_{t-1}+\varepsilon_{t}
\end{array}\right.
$$

with $\alpha_{t}, \beta_{t}$ as in (2.8). This is another expectations feedback system with expectation feedback from inflation forecasting. Realized inflations influence the beliefs agents have about economic reality and these beliefs feed back into the actual dynamics of economy and determine the future realized inflations together with an exogenous driving variable output gap or real marginal costs.

We further check the relationship between stability of $\operatorname{SCEE}\left(\alpha^{*}, \beta^{*}\right)$ and SAC-learning. For $\alpha^{*}$, since $0 \leq \frac{\lambda\left(1-\beta^{2}\right)}{1-\lambda \beta^{2}}<1$, it can be seen from (4.7) that $\alpha^{*}$ is stable. For $\beta^{*}$, because 


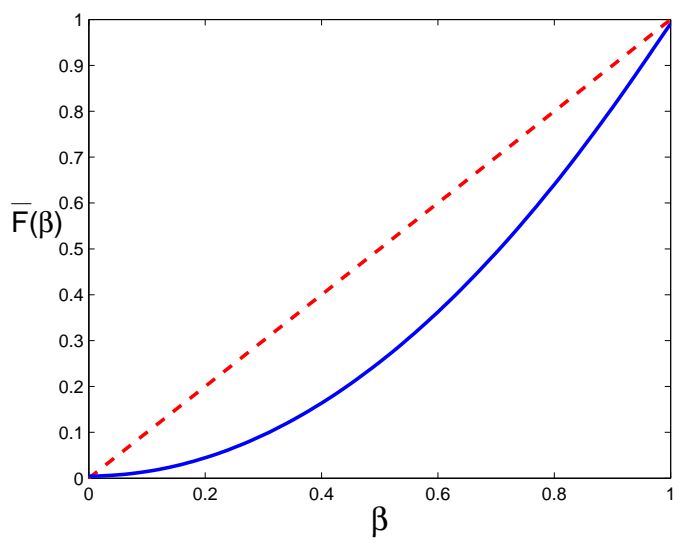

(a) $\gamma=0.01$

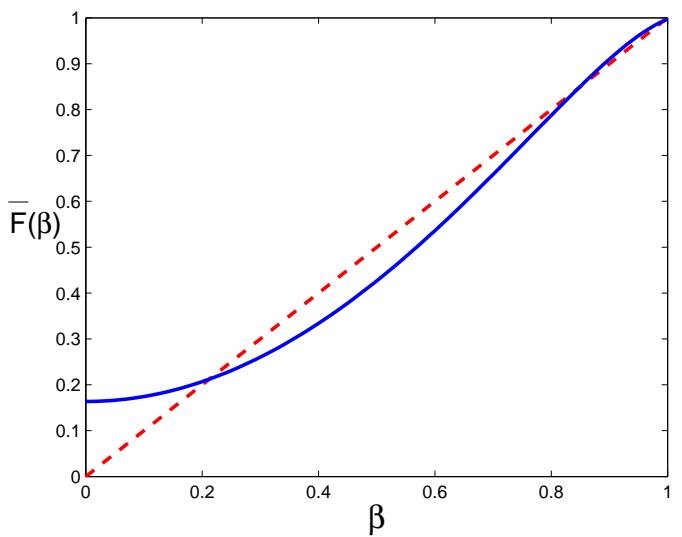

(c) $\gamma=0.065$

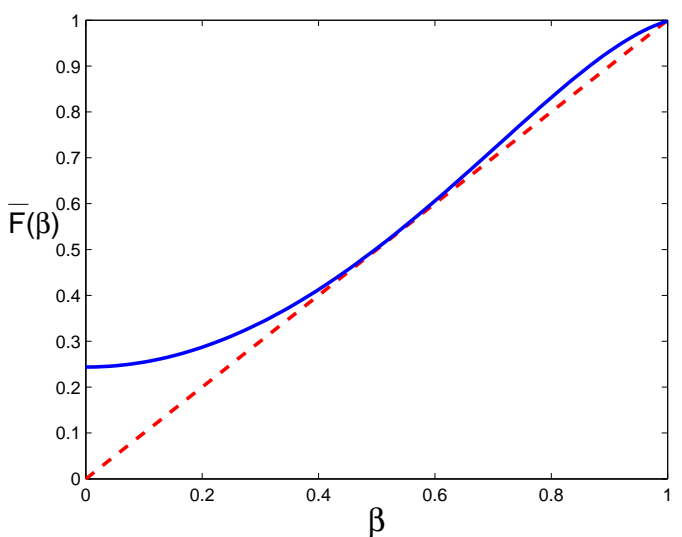

(e) $\gamma=0.084$

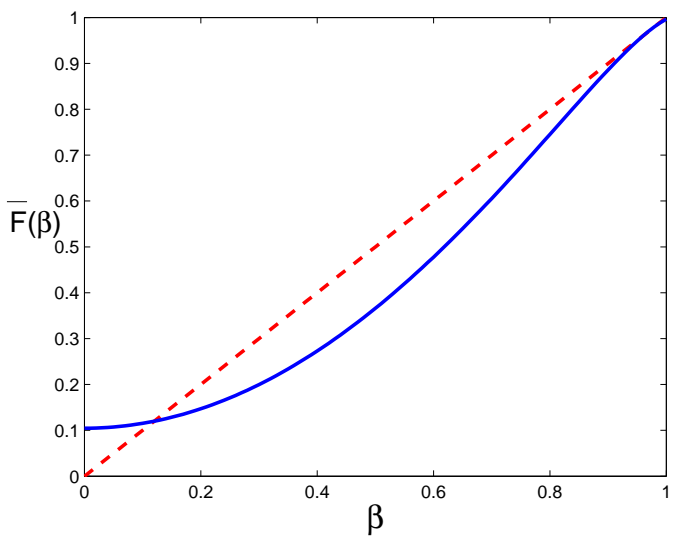

(b) $\gamma=0.05$

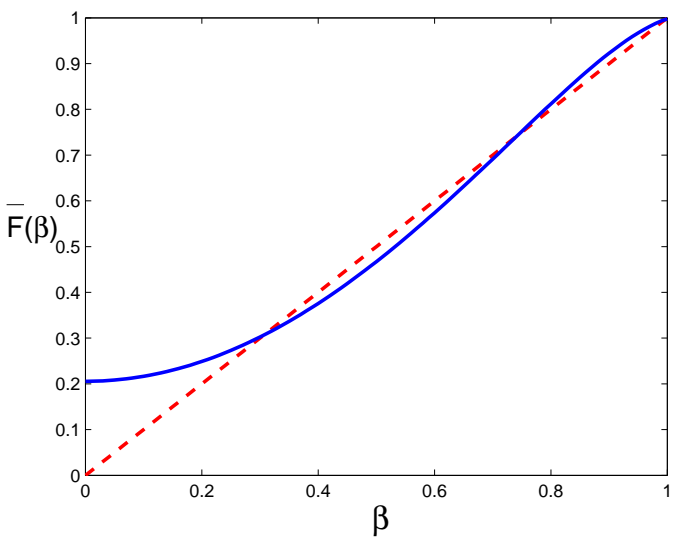

(d) $\gamma=0.075$

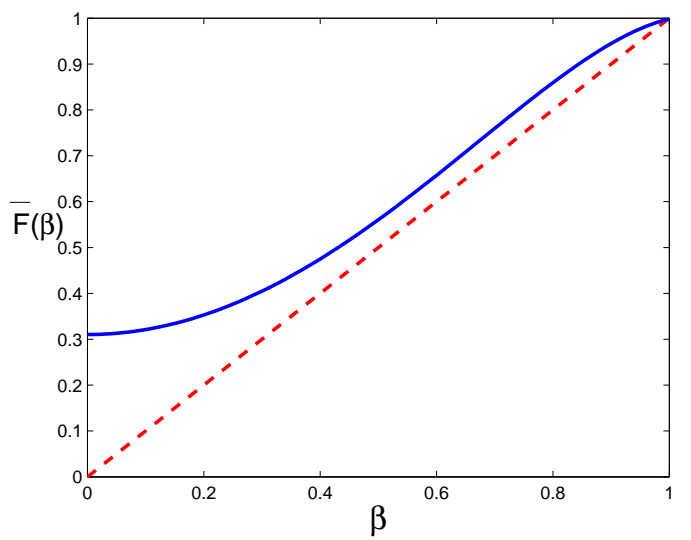

(f) $\gamma=0.1$

Figure 6: SCEE $\beta^{*}$ with $\gamma=0.01$ (a); $\gamma=0.05$ (b); $\gamma=0.065$ (c); $\gamma=0.075$ (d); $\gamma=0.084$ (e) and $\gamma=0.1(\mathrm{f})$, where $\frac{\sigma_{u}^{2}}{\sigma_{\varepsilon}^{2}}=0.1, \rho=0.9, \lambda=0.99$. 
of the complexity of the first-order autocorrelation $\bar{F}(\beta)$ in (4.8), it is difficult to check the stability of SCEE, or even the number of SCEE. We have the following relationship between the SCEE and the SAC-learning.

Proposition 4 If $(0 \leq) \bar{F}^{\prime}\left(\beta^{*}\right)<1$, then the SCEE $\left(\alpha^{*}, \beta^{*}\right)$ is stable, that is, the $S A C$ learning $\left(\alpha_{t}, \beta_{t}\right)$ converges to the SCEE $\left(\alpha^{*}, \beta^{*}\right)$ as time $t$ tends to $\infty$.

The proof is given in Appendix H. If the stable SCEE is not unique, the convergence depends on initial states of the system, as illustrated in the following numerical simulations.

\subsubsection{Numerical analysis}

For $\left(\pi_{0}, y_{0}\right)=(0.028,0.01)$, Figures $7 \mathrm{a}$ and $7 \mathrm{~b}$ show that the SAC-learning dynamics $\left(\alpha_{t}, \beta_{t}\right)$ converges to the stable low-persistence $\operatorname{SCEE}\left(\alpha^{*}, \beta^{*}\right)=(0.03,0.3066)$. Figure $7 \mathrm{a}$ illustrates that the mean of inflation $\alpha_{t}$ tends to the mean $\alpha^{*}=0.03$. Figure $7 \mathrm{~b}$ illustrates that the first-order autocorrelation coefficient of inflation $\beta_{t}$ slowly tends to the low-persistence stable first-order autocorrelation coefficient $\beta^{*}=0.3066$. For the different initial value $\left(\pi_{0}, y_{0}\right)=(0.1,0.15)$, our numerical simulation shows that the mean of inflation $\alpha_{t}$ under SAC-learning still tends to the mean $\alpha^{*}$, but slowly ${ }^{9}$ (see Figure $7 \mathrm{c}$ ), while Figure $7 \mathrm{~d}$ indicates that the first-order autocorrelation coefficient of inflation $\beta_{t}$ under SAC-learning tends to the higher stable first-order autocorrelation coefficient $\beta^{*}=0.9961^{10}$. Correspondingly given the same sample path of noise, the time series of inflation under SAC-learning can also replicate the time series of inflation in the SCEE after long-term learning as shown in the preceding asset pricing model.

Numerous simulations show that as initial values of inflation are (relatively) higher than the mean $\alpha^{*}=0.03$, the sample autocorrelation learning $\beta_{t}$ generally enters the high-persistence region. In particular, a large shock to the inflation may easily cause a jump of the SAC-learning process into the high-persistence region.

\section{Conclusion}

In this paper we have introduced the concept of behavioral learning equilibrium, a very simple type of misspecification equilibrium together with an intuitive behavioral

\footnotetext{
${ }^{9}$ The slow convergence is caused by the slope $\frac{\lambda-\lambda \beta^{2}}{1-\lambda \beta^{2}}$ in the expression (4.7), which is very close to 1 for $\lambda=0.99$, as shown in Figure 4a.

${ }^{10}$ As shown in Figure $4 \mathrm{~b}, \bar{F}^{\prime}\left(\beta^{*}\right)$ is close to 1 and hence the convergence of SAC-learning is very slow.
} 


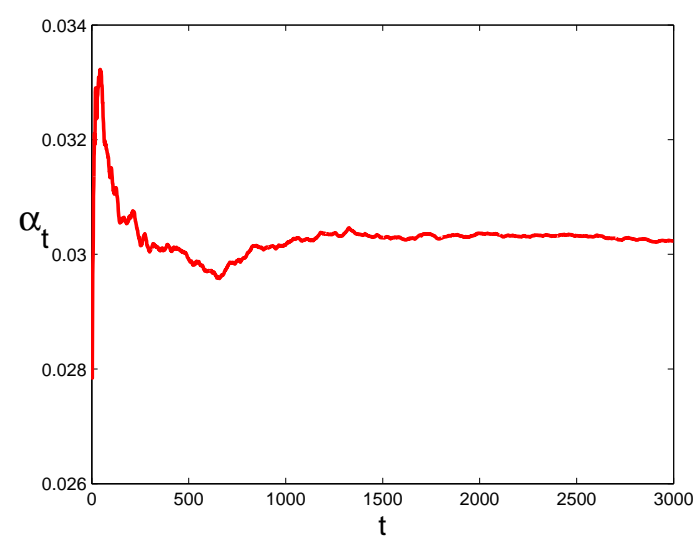

(a) $\alpha_{t} \rightarrow 0.03$

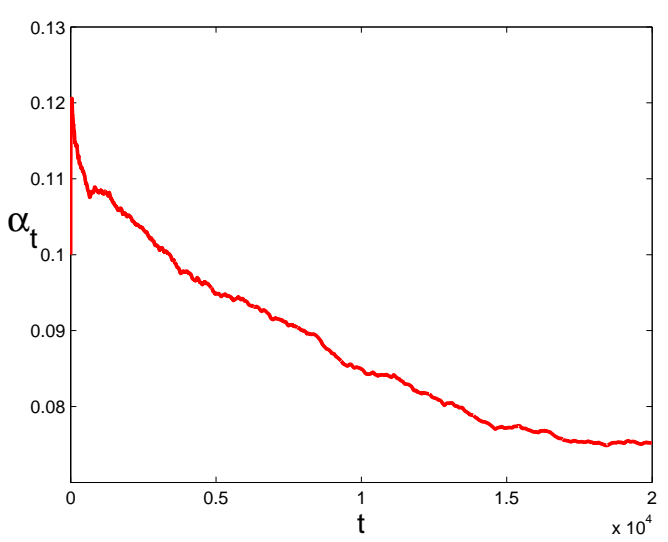

(c) $\alpha_{t} \rightarrow 0.03$

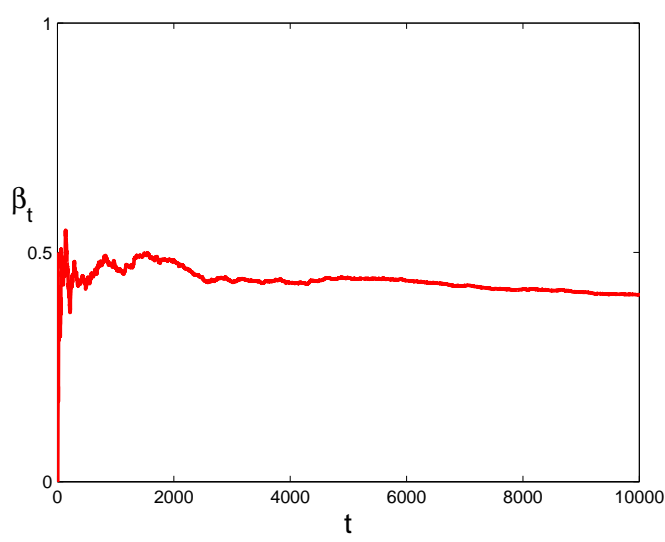

(b) $\beta_{t} \rightarrow 0.3066$

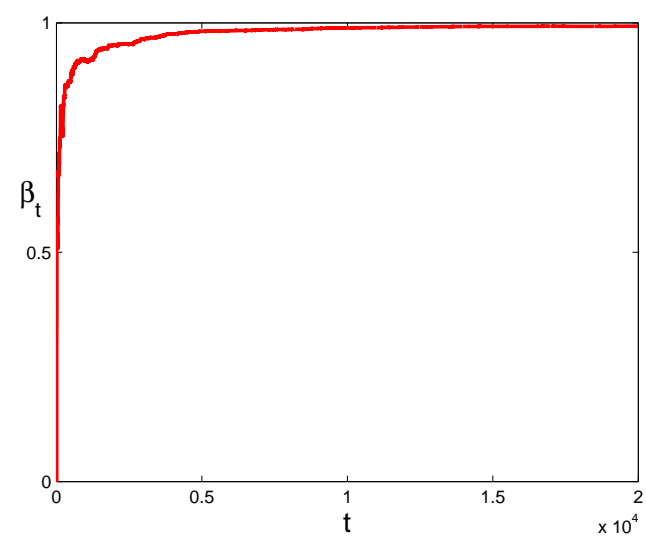

(d) $\beta_{t} \rightarrow 0.9961$

Figure 7: Time series of $\alpha_{t}$ and $\beta_{t}$ under SAC-learning with different initial values $\left(\pi_{0}, y_{0}\right)=(0.028,0.01)(\mathrm{a}),(\mathrm{b})$ and $\left(\pi_{0}, y_{0}\right)=(0.1,0.15)(\mathrm{c}),(\mathrm{d})$. 
learning process. Boundedly rational agents use a univariate linear forecasting rule and in equilibrium correctly forecast the unconditional sample mean and first-order sample autocorrelation. Hence, to a first order approximation the simple linear forecasting rule is consistent with observed market realizations. Sample autocorrelation learning simply means that agents are slowly updating the two coefficients -sample mean and first-order autocorrelation- of their linear rule. In the long run, agents thus learn the best univariate linear forecasting rule, without fully recognizing the structure of the economy.

We have applied our behavioral learning equilibrium concept to a standard asset pricing model with $\mathrm{AR}(1)$ dividends and a New Keynesian Philips curve driven by an $\mathrm{AR}(1)$ process for the output gap or marginal costs. In both applications, the law of motion of the economy is linear, but it is driven by an exogenous stochastic AR(1) process. Agents however are not fully aware of the exact linear structure of the economy, but use a simple univariate forecasting rule, to predict asset prices or inflation. In the asset pricing model a unique SCEE exists and it is globally stable under SAC-learning. An important feature of the SCEE is that it is characterized by high-persistence and excess volatility in asset prices, significantly higher than under rational expectations. In the New Keynesian model, multiple SCEE arise and a low and a high-persistence misspecification equilibrium co-exist. The SAC-learning exhibits path dependence and it depends on the initial states whether the system converges to the low-persistence or the high-persistence inflation regime. In particular, when there are shocks- e.g. oil shocks- temporarily causing high inflation, SAC-learning may lock into the high-persistence inflation regime.

Are these behavioral learning equilibria empirically relevant or would smart agents recognize their (second order) mistakes and learn to be perfectly rational? This empirical question should be addressed in more detail in future work, but we provide some arguments for the empirical relevance of our equilibrium concept. Firstly, in our applications the SCEE already explain some important stylized facts: (i) high persistence (close to unit root) and excess volatility in asset prices, (ii) high persistence in inflation and (iii) regime switching in inflation dynamics, which could explain a long phase of high US inflation in the 1970s and early 1980s as well as a long phase of low inflation in the 1990s and 2000s. Secondly, we stress the simplicity and behavioral interpretation of our learning equilibrium concept. The univariate $\mathrm{AR}(1)$ rule and the SAC-learning process are examples of simple forecasting heuristics that can be used without any knowledge of statistical techniques, simply by observing a time series and roughly "guestimating" its sample average and its first-order persistence coefficient. Coordination on a behavioral forecasting 
heuristic that performs reasonably well to a first-order approximation seems more likely than coordination on more complicated learning or sunspot equilibria, cf., for example, Woodford (1990). Even though some smart individual agents might be able to improve upon the best linear, univariate forecasting rule, a majority of agents might still stick to their simple univariate rule. It therefore seems relevant to describe aggregate phenomena by simple misspecification equilibria and behavioral learning processes. Our behavioral learning equilibrium concept also relates to the "natural expectations" in Fuster et al. (2010), emphasizing parsimonious forecasting rules giving much weight to recent changes to explain the long-run persistence of economic shocks. Our simple univariate AR(1) rule may be seen as the most parsimonious forecasting rule leading to long-run persistence. There is in fact already some experimental evidence for the relevance of misspecification equilibria in Adam (2007). More recently Assenza et al. (2011) and Pfajfar and Zakelj (2010) ran learning to forecasting experiments with human subjects in a New Keynesian framework with expectations feedback from individual inflation and output gap forecasts. Simple linear univariate models explain a substantial part of individual inflation and output gap forecasting behavior.

In future work we plan to consider more general economic settings and study behavioral learning equilibria. An obvious next step is to apply our SCEE and SAC-learning framework to higher dimensional linear economic systems, with agents forecasting by univariate linear rules. In particular, the fully specified New Keynesian model of inflation and output dynamics would be an interesting (two-dimensional) application. Finally, it is interesting and challenging to study SCEE and misspecification under heterogeneous expectations and allow for switching between different rules. Branch (2004) and Hommes (2011) provide some empirical and experimental evidence on heterogeneous expectations, while Berardi (2007) and Branch and Evans (2006, 2007) have made some related studies on heterogeneous expectations and learning in similar settings.

\section{Acknowledgements}

We would like to thank Kevin Lansing, Mikhail Anufriev, Michael Reiter, Florian Wagener, Cees Diks and other participants in the KAFEE lunch seminar at the University of Amsterdam, March 2011, the 3rd POLHIA workshop in Universita Politecnica delle Marche, Ancona, May 2010, the 11th Workshop on Optimal Control, Dynamic Games and Nonlinear Dynamics at the University of Amsterdam, June 2010 and the CEF 2011 
Conference, San Francisco, June 2011 for helpful comments. We are grateful to the EU 7th framework collaborative project "Monetary, Fiscal and Structural Policies with Heterogeneous Agents (POLHIA)", grant no. 225408, for financial support. Mei Zhu also acknowledges financial support from NSFC(10871005).

\section{Appendix}

\section{A Recursive dynamics of SAC-learning}

The sample average is

$$
\begin{aligned}
\alpha_{t} & =\frac{1}{t+1}\left[x_{0}+x_{1}+\cdots+x_{t}\right] \\
& =\frac{1}{t+1}\left[(t+1) \alpha_{t-1}+x_{t}-\alpha_{t-1}\right] \\
& =\alpha_{t-1}+\frac{1}{t+1}\left[x_{t}-\alpha_{t-1}\right] .
\end{aligned}
$$

Let

$$
\begin{aligned}
z_{t}:= & \left(x_{0}-\alpha_{t}\right)\left(x_{1}-\alpha_{t}\right)+\cdots+\left(x_{t-1}-\alpha_{t}\right)\left(x_{t}-\alpha_{t}\right) \\
= & \left(x_{0}-\alpha_{t-1}-\frac{1}{t+1}\left(x_{t}-\alpha_{t-1}\right)\right)\left(x_{1}-\alpha_{t-1}-\frac{1}{t+1}\left(x_{t}-\alpha_{t-1}\right)\right)+ \\
& \cdots+\left(x_{t-1}-\alpha_{t-1}-\frac{1}{t+1}\left(x_{t}-\alpha_{t-1}\right)\right)\left(x_{t}-\alpha_{t-1}-\frac{1}{t+1}\left(x_{t}-\alpha_{t-1}\right)\right) \\
= & \left(x_{0}-\alpha_{t-1}\right)\left(x_{1}-\alpha_{t-1}\right)+\cdots+\left(x_{t-2}-\alpha_{t-1}\right)\left(x_{t-1}-\alpha_{t-1}\right) \\
& +\frac{x_{t}-\alpha_{t-1}}{t+1}\left(2 \alpha_{t-1}-x_{0}-x_{1}+\cdots+2 \alpha_{t-1}-x_{t-2}-x_{t-1}\right)+\frac{t-1}{(t+1)^{2}}\left(x_{t}-\alpha_{t-1}\right)^{2} \\
& +\frac{t}{t+1}\left(x_{t-1}-\alpha_{t-1}\right)\left(x_{t}-\alpha_{t-1}\right)-\frac{t}{(t+1)^{2}}\left(x_{t}-\alpha_{t-1}\right)^{2} \\
= & z_{t-1}+\frac{1}{t+1}\left(x_{t}-\alpha_{t-1}\right)\left[2(t-1) \alpha_{t-1}-x_{0}-2 x_{1}-\cdots-2 x_{t-2}-x_{t-1}+t\left(x_{t-1}-\alpha_{t-1}\right)\right] \\
& -\frac{1}{(t+1)^{2}}\left(x_{t}-\alpha_{t-1}\right)^{2}, \\
= & z_{t-1}+\frac{1}{t+1}\left(x_{t}-\alpha_{t-1}\right)\left[x_{0}+(t+1) x_{t-1}-(t+2) \alpha_{t-1}\right]-\frac{1}{(t+1)^{2}}\left(x_{t}-\alpha_{t-1}\right)^{2} \\
= & z_{t-1}+\left(x_{t}-\alpha_{t-1}\right)\left[x_{t-1}+\frac{x_{0}}{t+1}-\frac{t+2}{t+1} \alpha_{t-1}+\frac{1}{(t+1)^{2}} \alpha_{t-1}-\frac{1}{(t+1)^{2}} x_{t}\right] \\
= & z_{t-1}+\left(x_{t}-\alpha_{t-1}\right) \Phi_{4},
\end{aligned}
$$


where $\Phi_{4}=x_{t-1}+\frac{x_{0}}{t+1}-\frac{t^{2}+3 t+1}{(t+1)^{2}} \alpha_{t-1}-\frac{1}{(t+1)^{2}} x_{t}$.

Write

$$
\begin{aligned}
n_{t} & :=\left(x_{0}-\alpha_{t}\right)^{2}+\left(x_{1}-\alpha_{t}\right)^{2}+\cdots+\left(x_{t}-\alpha_{t}\right)^{2} \\
& =\left(x_{0}-\alpha_{t-1}-\frac{1}{t+1}\left(x_{t}-\alpha_{t-1}\right)\right)^{2}+\cdots+\left(x_{t}-\alpha_{t-1}-\frac{1}{t+1}\left(x_{t}-\alpha_{t-1}\right)\right)^{2} \\
& =\left(x_{0}-\alpha_{t-1}\right)^{2}+\left(x_{1}-\alpha_{t-1}\right)^{2}+\cdots+\left(x_{t-1}-\alpha_{t-1}\right)^{2}+\frac{t+t^{2}}{(t+1)^{2}}\left(x_{t}-\alpha_{t-1}\right)^{2} \\
& =n_{t-1}+\frac{t}{t+1}\left(x_{t}-\alpha_{t-1}\right)^{2} .
\end{aligned}
$$

All these results are consistent with those in Appendix 1 of Hommes, Sorger \& Wagener (2004). Note that in our paper $R_{t}$ is different from $n_{t}$ in Hommes et al. (2004). In fact,

$$
\begin{aligned}
R_{t} & =\frac{1}{t+1} n_{t} \\
& =\frac{1}{t+1}\left[n_{t-1}+\frac{t}{t+1}\left(x_{t}-\alpha_{t-1}\right)^{2}\right] \\
& =\frac{t}{t+1} R_{t-1}+\frac{t}{(t+1)^{2}}\left(x_{t}-\alpha_{t-1}\right)^{2} \\
& =R_{t-1}+\frac{1}{t+1}\left[\frac{t}{t+1}\left(x_{t}-\alpha_{t-1}\right)^{2}-R_{t-1}\right] .
\end{aligned}
$$

Furthermore,

$$
\begin{aligned}
\beta_{t} & =\frac{z_{t}}{n_{t}} \\
& =\beta_{t-1}+\frac{1}{n_{t} n_{t-1}}\left[z_{t} n_{t-1}-z_{t-1} n_{t}\right] \\
& =\beta_{t-1}+\frac{1}{n_{t} n_{t-1}}\left[\left(z_{t-1}+\left(x_{t}-\alpha_{t-1}\right) \Phi_{4}\right) n_{t-1}-z_{t-1}\left(n_{t-1}+\frac{t}{t+1}\left(x_{t}-\alpha_{t-1}\right)^{2}\right)\right] \\
& =\beta_{t-1}+\frac{1}{n_{t} n_{t-1}}\left[\left(x_{t}-\alpha_{t-1}\right) \Phi_{4} n_{t-1}-z_{t-1} \frac{t}{t+1}\left(x_{t}-\alpha_{t-1}\right)^{2}\right] \\
& =\beta_{t-1}+\frac{1}{n_{t}}\left[\left(x_{t}-\alpha_{t-1}\right) \Phi_{4}-\beta_{t-1} \frac{t}{t+1}\left(x_{t}-\alpha_{t-1}\right)^{2}\right] \\
& =\beta_{t-1}+\frac{R_{t}^{-1}}{t+1}\left[\left(x_{t}-\alpha_{t-1}\right)\left(x_{t-1}+\frac{x_{0}}{t+1}-\frac{t^{2}+3 t+1}{(t+1)^{2}} \alpha_{t-1}-\frac{x_{t}}{(t+1)^{2}}\right)-\frac{t}{t+1} \beta_{t-1}\left(x_{t}-\alpha_{t-1}\right)^{2}\right]
\end{aligned}
$$




\section{B Rational expectations equilibrium of prices}

Under the assumption that the transversality condition $\lim _{k \rightarrow \infty} \frac{E_{t}\left(p_{t+k}\right)}{R^{k}}=0$ holds, the REE price can be computed as

$$
\begin{aligned}
p_{t}^{*} & =\frac{1}{R}\left[E_{t} p_{t+1}^{*}+E_{t} y_{t+1}\right] \\
& =\frac{a+\rho y_{t}}{R}+\frac{1}{R} E_{t} p_{t+1}^{*} \\
& =\frac{a+\rho y_{t}}{R}+\frac{1}{R} E_{t}\left\{\frac{1}{R}\left[E_{t+1} p_{t+2}^{*}+E_{t+1} y_{t+2}\right]\right\} \\
& =\frac{a+\rho y_{t}}{R}+\frac{E_{t}\left(a+\rho y_{t+1}\right)}{R^{2}}+\frac{1}{R^{2}} E_{t} p_{t+2}^{*} \\
& =\frac{a+\rho y_{t}}{R}+\frac{a+\rho a+\rho^{2} y_{t}}{R^{2}}+\frac{1}{R^{2}} E_{t} p_{t+2}^{*} \\
& =\cdots \\
& =\frac{a+\rho y_{t}}{R}+\frac{a+\rho a+\rho^{2} y_{t}}{R^{2}}+\cdots+\frac{a+\rho a+\cdots+\rho^{n-1} a+\rho^{n} y_{t}}{R^{n}}+\cdots \\
& =\frac{a+\rho y_{t}}{R}+\frac{a+\rho a+\rho^{2} y_{t}}{R^{2}}+\cdots+\frac{\frac{a\left(1-\rho^{n}\right)}{1-\rho}+\rho^{n} y_{t}}{R^{n}}+\cdots \\
& =\sum_{n=1}^{\infty} \frac{a}{1-\rho}\left[\left(\frac{1}{R}\right)^{n}-\left(\frac{\rho}{R}\right)^{n}\right]+\sum_{n=1}^{\infty}\left(\frac{\rho}{R}\right)^{n} y_{t} \\
& =\frac{a}{1-\rho}\left[\frac{1}{R-1}-\frac{\rho}{R-\rho}\right]+\frac{\rho}{R-\rho} y_{t} \\
& =\frac{a R}{(R-1)(R-\rho)}+\frac{\rho}{R-\rho} y_{t} .
\end{aligned}
$$

\section{First-order autocorrelation coefficient of price}

We rewrite (3.10) as

$$
p_{t}-\bar{p}=\frac{\beta^{2}}{R}\left(p_{t-1}-\bar{p}\right)+\frac{\rho}{R} \varepsilon_{t}+\frac{\rho^{2}}{R} \varepsilon_{t-1}+\cdots .
$$

Thus

$$
\begin{gathered}
E\left[\left(p_{t}-\bar{p}\right)\left(p_{t-1}-\bar{p}\right)\right]=E\left[\frac{\beta^{2}}{R}\left(p_{t-1}-\bar{p}\right)^{2}+\frac{\rho}{R} \varepsilon_{t}\left(p_{t-1}-\bar{p}\right)+\frac{\rho^{2}}{R} \varepsilon_{t-1}\left(p_{t-1}-\bar{p}\right)+\cdots\right] \\
=\frac{\beta^{2}}{R} E\left(p_{t-1}-\bar{p}\right)^{2}+0+\frac{\rho^{2}}{R} E\left[\varepsilon_{t-1}\left(p_{t-1}-\bar{p}\right)\right]+\cdots \\
E\left[\left(p_{t}-\bar{p}\right)^{2}\right] \\
=E\left[\frac{\beta^{2}}{R}\left(p_{t-1}-\bar{p}\right)\left(p_{t}-\bar{p}\right)+\frac{\rho}{R} \varepsilon_{t}\left(p_{t}-\bar{p}\right)+\frac{\rho^{2}}{R} \varepsilon_{t-1}\left(p_{t}-\bar{p}\right)+\cdots\right] \\
=\frac{\beta^{2}}{R} E\left[\left(p_{t-1}-\bar{p}\right)\left(p_{t}-\bar{p}\right)\right]+\frac{\rho}{R} E\left[\varepsilon_{t}\left(p_{t}-\bar{p}\right)\right]+\frac{\rho^{2}}{R} E\left[\varepsilon_{t-1}\left(p_{t}-\bar{p}\right)\right]+\cdots
\end{gathered}
$$


Thus based on (C.2) and (C.3),

$$
\begin{aligned}
E\left[\left(p_{t}-\bar{p}\right)^{2}\right]= & \frac{\beta^{2}}{R}\left[\frac{\beta^{2}}{R} E\left[\left(p_{t}-\bar{p}\right)^{2}\right]+\frac{\rho^{2}}{R} E\left[\varepsilon_{t}\left(p_{t}-\bar{p}\right)\right]+\frac{\rho^{3}}{R} E\left[\varepsilon_{t-1}\left(p_{t}-\bar{p}\right)\right]+\cdots\right] \\
& +\frac{\rho}{R} E\left[\varepsilon_{t}\left(p_{t}-\bar{p}\right)\right]+\frac{\rho^{2}}{R} E\left[\varepsilon_{t-1}\left(p_{t}-\bar{p}\right)\right]+\cdots \\
= & \left(\frac{\beta^{2}}{R}\right)^{2} E\left[\left(p_{t}-\bar{p}\right)^{2}\right]+\frac{\rho\left(\beta^{2} \rho+R\right)}{R^{2}}\left\{E\left[\varepsilon_{t}\left(p_{t}-\bar{p}\right)\right]+\rho E\left[\varepsilon_{t-1}\left(p_{t}-\bar{p}\right)\right]+\cdots\right\} .
\end{aligned}
$$

That is,

$$
E\left[\left(p_{t}-\bar{p}\right)^{2}\right]=\frac{\rho\left(\beta^{2} \rho+R\right)}{R^{2}-\beta^{4}}\left\{E\left[\varepsilon_{t}\left(p_{t}-\bar{p}\right)\right]+\rho E\left[\varepsilon_{t-1}\left(p_{t}-\bar{p}\right)\right]+\cdots\right\} .
$$

In the following we will calculate $E\left(\varepsilon_{t-k} p_{t}\right), k=0,1,2, \cdots$.

$$
\begin{aligned}
E\left[\varepsilon_{t}\left(p_{t}-\bar{p}\right)\right] & =\frac{\beta^{2}}{R} E\left[\left(p_{t-1}-\bar{p}\right) \varepsilon_{t}\right]+\frac{\rho}{R} E\left[\varepsilon_{t}^{2}\right]+\frac{\rho^{2}}{R} E\left[\varepsilon_{t-1} \varepsilon_{t}\right]+\cdots \\
& =\frac{\rho}{R} \sigma_{\varepsilon}^{2} . \\
E\left[\varepsilon_{t-1}\left(p_{t}-\bar{p}\right)\right] & =\frac{\beta^{2}}{R} E\left[\left(p_{t-1}-\bar{p}\right) \varepsilon_{t-1}\right]+\frac{\rho}{R} E\left[\varepsilon_{t} \varepsilon_{t-1}\right]+\frac{\rho^{2}}{R} E\left[\varepsilon_{t-1}^{2}\right]+\cdots \\
& =\frac{\beta^{2}}{R} E\left[\left(p_{t}-\bar{p}\right) \varepsilon_{t}\right]+\frac{\rho^{2}}{R} \sigma_{\varepsilon}^{2} \\
& =\frac{\beta^{2}}{R} \cdot \frac{\rho}{R} \sigma_{\varepsilon}^{2}+\frac{\rho^{2}}{R} \sigma_{\varepsilon}^{2} .
\end{aligned}
$$

$$
\begin{aligned}
E\left[\varepsilon_{t-2}\left(p_{t}-\bar{p}\right)\right]= & \frac{\beta^{2}}{R} E\left[\left(p_{t-1}-\bar{p}\right) \varepsilon_{t-2}\right]+\frac{\rho}{R} E\left[\varepsilon_{t} \varepsilon_{t-2}\right]+\frac{\rho^{2}}{R} E\left[\varepsilon_{t-1} \varepsilon_{t-2}\right]+\frac{\rho^{3}}{R} E\left[\varepsilon_{t-2}^{2}\right]+\cdots \\
= & \frac{\beta^{2}}{R} E\left[\left(p_{t}-\bar{p}\right) \varepsilon_{t-1}\right]+\frac{\rho^{3}}{R} \sigma_{\varepsilon}^{2} \\
= & \left(\frac{\beta^{2}}{R}\right)^{2} \frac{\rho}{R} \sigma_{\varepsilon}^{2}+\frac{\beta^{2}}{R} \cdot \frac{\rho^{2}}{R} \sigma_{\varepsilon}^{2}+\frac{\rho^{3}}{R} \sigma_{\varepsilon}^{2} \cdot \\
E\left[\varepsilon_{t-k}\left(p_{t}-\bar{p}\right)\right]= & \frac{\beta^{2}}{R} E\left[\left(p_{t-1}-\bar{p}\right) \varepsilon_{t-k}\right]+\frac{\rho}{R} E\left[\varepsilon_{t} \varepsilon_{t-k}\right]+\frac{\rho^{2}}{R} E\left[\varepsilon_{t-1} \varepsilon_{t-k}\right]+\cdots+\frac{\rho^{k+1}}{R} E\left[\varepsilon_{t-k}^{2}\right]+\cdots \\
= & \frac{\beta^{2}}{R} E\left[\left(p_{t}-\bar{p}\right) \varepsilon_{t-k+1}\right]+\frac{\rho^{k+1}}{R} \sigma_{\varepsilon}^{2} \\
= & \cdots \\
= & \frac{\rho}{R} \sigma_{\varepsilon}^{2} \cdot\left(\frac{\beta^{2}}{R}\right)^{k}+\frac{\rho}{R} \sigma_{\varepsilon}^{2} \cdot\left(\frac{\beta^{2}}{R}\right)^{k-1} \cdot \rho+\frac{\rho}{R} \sigma_{\varepsilon}^{2} \cdot\left(\frac{\beta^{2}}{R}\right)^{k-2} \cdot \rho^{2}+\cdots \\
& +\frac{\rho}{R} \sigma_{\varepsilon}^{2} \cdot \frac{\beta^{2}}{R} \cdot \rho^{k-1}+\frac{\rho}{R} \sigma_{\varepsilon}^{2} \cdot \rho^{k} \\
= & \frac{\sigma_{\varepsilon}^{2}}{R} \cdot \frac{\rho^{k+1}-\left(\frac{\beta^{2}}{R}\right)^{k+1}}{1-\frac{\beta^{2}}{\rho R}} .
\end{aligned}
$$


Hence

$$
\begin{aligned}
& E\left[\varepsilon_{t}\left(p_{t}-\bar{p}\right)\right]+\rho E\left[\varepsilon_{t-1}\left(p_{t}-\bar{p}\right)\right]+\cdots \\
= & \frac{\sigma_{\varepsilon}^{2}}{\rho R} \cdot \frac{\sum_{k=0}^{\infty} \rho^{2(k+1)}-\sum_{k=0}^{\infty}\left(\frac{\beta^{2} \rho}{R}\right)^{k+1}}{1-\frac{\beta^{2}}{\rho R}} \\
= & \frac{\sigma_{\varepsilon}^{2}}{\rho R} \cdot \frac{\frac{\rho^{2}}{1-\rho^{2}}-\frac{\beta^{2} \rho}{R-\rho \beta^{2}}}{1-\frac{\beta^{2}}{\rho R}} \\
= & \frac{\rho \sigma_{\varepsilon}^{2}}{\left(1-\rho^{2}\right)\left(R-\rho \beta^{2}\right)} .
\end{aligned}
$$

Substituting (C.5) into (C.4), we obtain

$$
\begin{aligned}
\operatorname{Var}\left(p_{t}\right) & =E\left[\left(p_{t}-\bar{p}\right)^{2}\right] \\
& =\frac{\rho\left(\beta^{2} \rho+R\right)}{R^{2}-\beta^{4}}\left\{E\left[\varepsilon_{t}\left(p_{t}-\bar{p}\right)\right]+\rho E\left[\varepsilon_{t-1}\left(p_{t}-\bar{p}\right)\right]+\cdots\right\} \\
& =\frac{\rho\left(\beta^{2} \rho+R\right)}{R^{2}-\beta^{4}} \cdot \frac{\rho \sigma_{\varepsilon}^{2}}{\left(1-\rho^{2}\right)\left(R-\rho \beta^{2}\right)} \\
& =\frac{\sigma_{\varepsilon}^{2} \rho^{2}\left(\beta^{2} \rho+R\right)}{\left(R^{2}-\beta^{4}\right)\left(1-\rho^{2}\right)\left(R-\rho \beta^{2}\right)} .
\end{aligned}
$$

Furthermore, based on (C.2),

$$
\begin{aligned}
\operatorname{Corr}\left(p_{t}, p_{t-1}\right) & =E\left[\left(p_{t}-\bar{p}\right)\left(p_{t-1}-\bar{p}\right)\right] / \operatorname{Var}\left(p_{t}\right) \\
& =\frac{\beta^{2}}{R}+\frac{\rho^{2}}{R \operatorname{Var}\left(p_{t}\right)}\left\{E\left[\varepsilon_{t}\left(p_{t}-\bar{p}\right)\right]+\rho E\left[\varepsilon_{t-1}\left(p_{t}-\bar{p}\right)\right]+\cdots\right\} \\
& =\frac{\beta^{2}}{R}+\frac{\rho^{2}}{R} \frac{\frac{\rho \sigma_{\varepsilon}^{2}}{\left(1-\rho^{2}\right)\left(R-\rho \beta^{2}\right)}}{\frac{\sigma_{\varepsilon}^{2} \rho^{2}\left(\rho \beta^{2}+R\right)}{\left(R^{2}-\beta^{4}\right)\left(1-\rho^{2}\right)\left(R-\rho \beta^{2}\right)}} \\
& =\frac{\beta^{2}}{R}+\frac{\rho\left(R^{2}-\beta^{4}\right)}{R\left(\rho \beta^{2}+R\right)} \\
& =\frac{\beta^{2}+R \rho}{\rho \beta^{2}+R} .
\end{aligned}
$$

\section{Proof of uniqueness of $\beta^{*}$ (Proposition 1)}

Using the first-order autocorrelation $F(\beta)$ in (3.13), it can be calculated that

$$
F^{\prime \prime}(\beta)=\frac{2 R\left(1-\rho^{2}\right)}{\left(\rho \beta^{2}+R\right)^{2}}-\frac{8 \rho \beta^{2} R\left(1-\rho^{2}\right)}{\left(\rho \beta^{2}+R\right)^{3}}=\frac{2 R\left(1-\rho^{2}\right)\left(R-3 \rho \beta^{2}\right)}{\left(\rho \beta^{2}+R\right)^{3}} .
$$

Therefore, if $\rho \leq \frac{R}{3}$, then $R-3 \beta^{2} \rho \geq R-\beta^{2} R>0$. Thus $G^{\prime \prime}(\beta)=F^{\prime \prime}(\beta)>0$. Note that $G(0)>0, G^{\prime}(0)=-1<0$ and $G(1)<0, G^{\prime}(1)=\frac{2 R\left(1-\rho^{2}\right)}{(\rho+R)^{2}}-1$. Hence if 
$G^{\prime}(1) \leq 0$, then $G^{\prime}\left(\beta^{*}\right)<0$. If $G^{\prime}(1)>0$, then there exists a minimal point $\beta_{1}$ such that $G^{\prime}\left(\beta_{1}\right)=0$. Moreover, since $G(1)<0$, then $G\left(\beta_{1}\right)<0$ (otherwise, $G(1) \geq G\left(\beta_{1}\right) \geq 0$, which is contradictory to $G(1)<0)$. Hence $\beta^{*}\left(\in\left(0, \beta_{1}\right)\right)$ is unique and $G^{\prime}\left(\beta^{*}\right)<0$, hence $0<F^{\prime}\left(\beta^{*}\right)<1$.

If $\rho>\frac{R}{3}$, then $\left.G^{\prime \prime}(\beta)\right|_{\beta=\sqrt{R /(3 \rho)}}=\left.F^{\prime \prime}(\beta)\right|_{\beta=\sqrt{R /(3 \rho)}}=0$ and $\left.G^{\prime}(\beta)\right|_{\beta=\sqrt{R /(3 \rho)}}$ is maximal. Thus in the case that $\rho>\frac{R}{3}$,

$$
\begin{aligned}
G^{\prime}(\beta) & =F^{\prime}(\beta)-1 \\
& =\frac{2 \beta R\left(1-\rho^{2}\right)}{\left(\rho \beta^{2}+R\right)^{2}}-1 \\
& <\frac{2 \sqrt{R /(3 \rho)} R\left(1-\rho^{2}\right)}{(R / 3+R)^{2}}-1 \\
& =\frac{3 \sqrt{3}\left(1-\rho^{2}\right)}{8 \sqrt{R \rho}}-1 \\
& <\frac{3 \sqrt{3}\left(1-R^{2} / 9\right)}{8 \sqrt{R^{2} / 3}}-1 \\
& =\frac{-(R-1)(R+9)}{8 R}<0 .
\end{aligned}
$$

That is, $G(\beta)$ is monotone. Therefore, in the case that $0<\rho<1, \beta^{*}$ is unique and $G^{\prime}\left(\beta^{*}\right)<0$, hence $0<F^{\prime}\left(\beta^{*}\right)<1$.

\section{E Proof of Proposition 2}

Under the SAC-learning defined in Section 2, the state dynamics equations become

$$
\left\{\begin{array}{l}
p_{t}=\frac{1}{R}\left[\alpha_{t-1}+\beta_{t-1}^{2}\left(p_{t-1}-\alpha_{t-1}\right)+a+\rho y_{t}\right], \\
y_{t}=a+\rho y_{t-1}+\varepsilon_{t} .
\end{array}\right.
$$

Set $\gamma_{t}=(1+t)^{-1}$. Since all functions are smooth, the learning rule (2.8) satisfies the conditions (A.1-A.3) of Section 6.2.1 in Evans and Honkapohja (2001, p.124).

In order to check the conditions (B.1-B.2) of Section 6.2.1 in Evans and Honkapohja (2001, p.125), we rewrite the system in matrix form by

$$
X_{t}=A\left(\theta_{t-1}\right) X_{t-1}+B\left(\theta_{t-1}\right) W_{t},
$$


where $\theta_{t}^{\prime}=\left(\alpha_{t}, \beta_{t}, R_{t}\right), X_{t}^{\prime}=\left(1, p_{t}, p_{t-1}, y_{t}\right)$ and $W_{t}^{\prime}=\left(1, \varepsilon_{t}\right)$,

$$
\begin{gathered}
A(\theta)=\left(\begin{array}{cccc}
0 & 0 & 0 & 0 \\
\frac{a(1+\rho)+\alpha\left(1-\beta^{2}\right)}{R} & \frac{\beta^{2}}{R} & 0 & \frac{\rho^{2}}{R} \\
0 & 1 & 0 & 0 \\
a & 0 & 0 & \rho
\end{array}\right), \\
B(\theta)=\left(\begin{array}{cc}
1 & 0 \\
0 & \frac{\rho}{R} \\
0 & 0 \\
0 & 1
\end{array}\right) .
\end{gathered}
$$

As shown in Evans and Honkapohja (2001, p.186), $A(\theta)$ and $B(\theta)$ satisfy the Lipschitz conditions and $B$ is bounded. Since $\varepsilon_{t}$ is assumed to have bounded moments, condition (B.1) is satisfied. Furthermore, the eigenvalues of matrix $A(\theta)$ are 0 (double), $\rho$ and $\frac{\beta^{2}}{R}$. According to the assumption $|\beta| \leq 1$ and $0<\rho<1$, all eigenvalues of $A(\theta)$ are less than 1 in absolute value. Then it follows that there is a compact neighborhood including the SCEE solution $\left(\alpha^{*}, \beta^{*}\right)$ on which the condition that $|A(\theta)|$ is bounded strictly below 1 is satisfied.

Thus the technical conditions for Section 6.2.1 of Chapter 6 in Evans and Honkapohja (2001) are satisfied. Moreover, since $p_{t}$ is stationary under the condition $|\beta| \leq 1$ and $0<\rho<1$, the limits

$$
\sigma^{2}:=\lim _{t \rightarrow \infty} E\left(p_{t}-\alpha\right)^{2}, \quad \sigma_{p p_{-1}}^{2}:=\lim _{t \rightarrow \infty} E\left(p_{t}-\alpha\right)\left(p_{t-1}-\alpha\right)
$$

exist and are finite. Hence according to Section 6.2 .1 of Chapter 6 in Evans and Honkapohja (2001, p.126), the associated ODE is

$$
\left\{\begin{array}{l}
\frac{d \alpha}{d \tau}=\bar{p}(\alpha, \beta)-\alpha \\
\frac{d \beta}{d \tau}=\sigma^{-2}\left[\sigma_{p p_{-1}}^{2}-\beta \sigma^{2}\right] \\
\frac{d R}{d \tau}=\sigma^{2}-R
\end{array}\right.
$$

That is,

$$
\left\{\begin{array}{l}
\frac{d \alpha}{d \tau}=\frac{\alpha\left(1-\beta^{2}\right)+\bar{y}}{R-\beta^{2}}-\alpha=\frac{-r \alpha+\bar{y}}{R-\beta^{2}} \\
\frac{d \beta}{d \tau}=F(\beta)-\beta=\frac{\beta^{2}+R \rho}{\rho \beta^{2}+R}-\beta .
\end{array}\right.
$$


Furthermore,

$$
J F\left(\alpha^{*}, \beta^{*}\right)=\left(\begin{array}{cc}
\frac{-r}{R-\left(\beta^{*}\right)^{2}} & 0 \\
0 & F^{\prime}\left(\beta^{*}\right)-1
\end{array}\right) .
$$

Based on the analysis in Appendix D, $F^{\prime}\left(\beta^{*}\right)-1<0$. Therefore, the unique stable $\operatorname{SCEE}\left(\alpha^{*}, \beta^{*}\right)$ corresponds to the unique stable fixed point of the ODE (E.2). Thus the SAC learning $\left(\alpha_{t}, \beta_{t}\right)$ converges to the unique stable $\operatorname{SCEE}\left(\alpha^{*}, \beta^{*}\right)$ as time $t$ tends to $\infty$.

\section{F Rational expectations equilibrium inflation}

Under the assumption that the transversality condition $\lim _{k \rightarrow \infty} \lambda^{k} E_{t}\left(\pi_{t+k}^{*}\right)=0$ holds, the REE inflation is computed as

$$
\begin{aligned}
\pi_{t}^{*} & =\lambda E_{t} \pi_{t+1}^{*}+\gamma y_{t}+u_{t} \\
& =\lambda E_{t}\left[\lambda E_{t+1} \pi_{t+2}^{*}+\gamma y_{t+1}+u_{t+1}\right]+\gamma y_{t}+u_{t} \\
& =\lambda^{2} E_{t} \pi_{t+2}^{*}+\gamma \lambda a+\gamma \lambda \rho y_{t}+\gamma y_{t}+u_{t} \\
& =\cdots \\
& =\sum_{k=1}^{\infty} \gamma \lambda^{k}\left(\rho^{k-1} a+\cdots+\rho a+a\right)+\sum_{k=0}^{\infty} \gamma(\lambda \rho)^{k} y_{t}+u_{t} \\
& =\sum_{k=1}^{\infty} \frac{\gamma a}{1-\rho}\left[\lambda^{k}-(\lambda \rho)^{k}\right]+\frac{\gamma}{1-\lambda \rho} y_{t}+u_{t} \\
& =\frac{\gamma \lambda a}{(1-\lambda)(1-\lambda \rho)}+\frac{\gamma}{1-\lambda \rho} y_{t}+u_{t} .
\end{aligned}
$$

\section{G First-order autocorrelation coefficient of inflation}

We rewrite model (4.6) as

$$
\left\{\begin{array}{l}
\pi_{t}-\bar{\pi}=\lambda \beta^{2}\left(\pi_{t-1}-\bar{\pi}\right)+\gamma\left(y_{t}-\bar{y}\right)+u_{t}, \\
y_{t}-\bar{y}=\rho\left(y_{t-1}-\bar{y}\right)+\varepsilon_{t}
\end{array}\right.
$$

That is,

$$
\left\{\begin{array}{l}
\pi_{t}-\bar{\pi}=\lambda \beta^{2}\left(\pi_{t-1}-\bar{\pi}\right)+\gamma \rho\left(y_{t-1}-\bar{y}\right)+\gamma \varepsilon_{t}+u_{t} \\
y_{t}-\bar{y}=\rho\left(y_{t-1}-\bar{y}\right)+\varepsilon_{t} .
\end{array}\right.
$$




$$
\begin{aligned}
& E\left[\left(\pi_{t}-\bar{\pi}\right)\left(\pi_{t-1}-\bar{\pi}\right)\right] \\
= & E\left[\lambda \beta^{2}\left(\pi_{t-1}-\bar{\pi}\right)^{2}+\gamma \rho\left(\pi_{t-1}-\bar{\pi}\right)\left(y_{t-1}-\bar{y}\right)+\gamma\left(\pi_{t-1}-\bar{\pi}\right) \varepsilon_{t}+\left(\pi_{t-1}-\bar{\pi}\right) u_{t}\right] \\
= & \lambda \beta^{2} \operatorname{Var}\left(\pi_{t}\right)+\gamma \rho E\left[\left(\pi_{t-1}-\bar{\pi}\right)\left(y_{t-1}-\bar{y}\right)\right]+\gamma E\left[\left(\pi_{t-1}-\bar{\pi}\right) \varepsilon_{t}\right]+\left(\pi_{t-1}-\bar{\pi}\right) u_{t} \\
= & \lambda \beta^{2} \operatorname{Var}\left(\pi_{t}\right)+\gamma \rho E\left[\left(\pi_{t-1}-\bar{\pi}\right)\left(y_{t-1}-\bar{y}\right)\right] \\
= & \lambda \beta^{2} \operatorname{Var}\left(\pi_{t}\right)+\gamma \rho E\left[\left(\pi_{t}-\bar{\pi}\right)\left(y_{t}-\bar{y}\right)\right] .
\end{aligned}
$$

$$
\begin{aligned}
& \operatorname{Var}\left(\pi_{t}\right) \\
= & E\left(\pi_{t}-\bar{\pi}\right)^{2} \\
= & E\left[\lambda \beta^{2}\left(\pi_{t}-\bar{\pi}\right)\left(\pi_{t-1}-\bar{\pi}\right)+\gamma \rho\left(\pi_{t}-\bar{\pi}\right)\left(y_{t-1}-\bar{y}\right)+\gamma\left(\pi_{t}-\bar{\pi}\right) \varepsilon_{t}+\left(\pi_{t}-\bar{\pi}\right) u_{t}\right] \\
= & \left.\lambda \beta^{2} E\left[\left(\pi_{t}-\bar{\pi}\right)\left(\pi_{t-1}-\bar{\pi}\right)\right]+\gamma \rho E\left[\left(\pi_{t}-\bar{\pi}\right)\left(y_{t-1}-\bar{y}\right)\right]+\gamma\left(\pi_{t}-\bar{\pi}\right) \varepsilon_{t}+\left(\pi_{t}-\bar{\pi}\right) u_{t}\right] \\
= & \lambda \beta^{2} E\left[\left(\pi_{t}-\bar{\pi}\right)\left(\pi_{t-1}-\bar{\pi}\right)\right]+\gamma \rho E\left[\left(\pi_{t}-\bar{\pi}\right)\left(y_{t-1}-\bar{y}\right)\right]+\gamma^{2} \sigma_{\varepsilon}^{2}+\sigma_{u}^{2},
\end{aligned}
$$

where the last equation is based on the fact that $E\left[\left(\pi_{t}-\bar{\pi}\right) \varepsilon_{t}\right]=E\left[\lambda \beta^{2}\left(\pi_{t-1}-\bar{\pi}\right) \varepsilon_{t}+\right.$ $\left.\gamma \rho\left(y_{t-1}-\bar{y}\right) \varepsilon_{t}+\gamma \varepsilon_{t}^{2}+u_{t} \varepsilon_{t}\right]=\gamma \sigma_{\varepsilon}^{2}$ and $E\left[\left(\pi_{t}-\bar{\pi}\right) u_{t}\right]=E\left[\lambda \beta^{2}\left(\pi_{t-1}-\bar{\pi}\right) u_{t}+\gamma \rho\left(y_{t-1}-\right.\right.$ $\left.\bar{y}) u_{t}+\gamma \varepsilon_{t} u_{t}+u_{t}^{2}\right]=\sigma_{u}^{2}$.

Based on (G.3) and (G.4),

$$
\begin{aligned}
\operatorname{Var}\left(\pi_{t}\right) & =\lambda \beta^{2} E\left[\left(\pi_{t}-\bar{\pi}\right)\left(\pi_{t-1}-\bar{\pi}\right)\right]+\gamma \rho E\left[\left(\pi_{t}-\bar{\pi}\right)\left(y_{t-1}-\bar{y}\right)\right]+\gamma^{2} \sigma_{\varepsilon}^{2}+\sigma_{u}^{2} \\
& =\lambda \beta^{2}\left[\lambda \beta^{2} \operatorname{Var}\left(\pi_{t}\right)+\gamma \rho E\left[\left(\pi_{t}-\bar{\pi}\right)\left(y_{t}-\bar{y}\right)\right]\right]+\gamma \rho E\left[\left(\pi_{t}-\bar{\pi}\right)\left(y_{t-1}-\bar{y}\right)\right]+\gamma^{2} \sigma_{\varepsilon}^{2}+\sigma_{u}^{2} \\
& =\lambda^{2} \beta^{4} \operatorname{Var}\left(\pi_{t}\right)+\lambda \beta^{2} \gamma \rho E\left[\left(\pi_{t}-\bar{\pi}\right)\left(y_{t}-\bar{y}\right)\right]+\gamma \rho E\left[\left(\pi_{t}-\bar{\pi}\right)\left(y_{t-1}-\bar{y}\right)\right]+\gamma^{2} \sigma_{\varepsilon}^{2}+\sigma_{u}^{2} .
\end{aligned}
$$

That is,

$$
\operatorname{Var}\left(\pi_{t}\right)=\frac{\lambda \beta^{2} \gamma \rho E\left[\left(\pi_{t}-\bar{\pi}\right)\left(y_{t}-\bar{y}\right)\right]+\gamma \rho E\left[\left(\pi_{t}-\bar{\pi}\right)\left(y_{t-1}-\bar{y}\right)\right]+\gamma^{2} \sigma_{\varepsilon}^{2}+\sigma_{u}^{2}}{1-\lambda^{2} \beta^{4}} .
$$

Thus, in order to obtain $E\left[\left(\pi_{t}-\bar{\pi}\right)\left(\pi_{t-1}-\bar{\pi}\right)\right]$ and $\operatorname{Var}\left(\pi_{t}\right)$, we need calculate $E\left[\left(\pi_{t}-\right.\right.$ $\left.\bar{\pi})\left(y_{t}-\bar{y}\right)\right]$ and $E\left[\left(\pi_{t}-\bar{\pi}\right)\left(y_{t-1}-\bar{y}\right)\right]$.

$$
\begin{aligned}
E\left[\left(\pi_{t}-\bar{\pi}\right)\left(y_{t}-\bar{\pi}\right)\right]= & E\left[\lambda \beta^{2}\left(\pi_{t-1}-\bar{\pi}\right)\left(y_{t}-\bar{y}\right)+\gamma \rho\left(y_{t-1}-\bar{\pi}\right)\left(y_{t}-\bar{y}\right)+\gamma \varepsilon_{t}\left(y_{t}-\bar{\pi}\right)+u_{t}\left(y_{t}-\bar{\pi}\right)\right] \\
= & \lambda \beta^{2} E\left\{\left(\pi_{t-1}-\bar{\pi}\right)\left[\rho\left(y_{t-1}-\bar{y}\right)+\varepsilon_{t}\right]\right\}+\gamma \rho E\left[\left(y_{t-1}-\bar{\pi}\right)\left(y_{t}-\bar{y}\right)\right] \\
& +\gamma E\left\{\varepsilon_{t}\left[\rho\left(y_{t-1}-\bar{y}\right)+\varepsilon_{t}\right]\right\}+E\left[u_{t}\left(y_{t}-\bar{\pi}\right)\right] \\
= & \lambda \beta^{2} \rho E\left[\left(\pi_{t-1}-\bar{\pi}\right)\left(y_{t-1}-\bar{\pi}\right)\right]+0+\frac{\gamma \rho^{2} \sigma_{\varepsilon}^{2}}{\left(1-\rho^{2}\right)}+\gamma \sigma_{\varepsilon}^{2}+0 .
\end{aligned}
$$


Thus

$$
E\left[\left(\pi_{t}-\bar{\pi}\right)\left(y_{t}-\bar{\pi}\right)\right]=\frac{\gamma \sigma_{\varepsilon}^{2}}{\left(1-\rho^{2}\right)\left(1-\lambda \beta^{2} \rho\right)} .
$$

Hence based on (G.6),

$$
\begin{aligned}
& E\left[\left(\pi_{t}-\bar{\pi}\right)\left(y_{t-1}-\bar{\pi}\right)\right] \\
= & E\left[\lambda \beta^{2}\left(\pi_{t-1}-\bar{\pi}\right)\left(y_{t-1}-\bar{y}\right)+\gamma \rho\left(y_{t-1}-\bar{\pi}\right)^{2}+\gamma \varepsilon_{t}\left(y_{t-1}-\bar{\pi}\right)+u_{t}\left(y_{t-1}-\bar{\pi}\right)\right] \\
= & \lambda \beta^{2} E\left[\left(\pi_{t-1}-\bar{\pi}\right)\left(y_{t-1}-\bar{y}\right)\right]+\gamma \rho E\left(y_{t-1}-\bar{\pi}\right)^{2}+0+0 \\
= & \lambda \beta^{2} \cdot \frac{\gamma \sigma_{\varepsilon}^{2}}{\left(1-\rho^{2}\right)\left(1-\lambda \beta^{2} \rho\right)}+\gamma \rho \cdot \frac{\sigma_{\varepsilon}^{2}}{1-\rho^{2}} \\
= & \frac{\gamma \sigma_{\varepsilon}^{2}}{\left(1-\rho^{2}\right)}\left[\frac{\lambda \beta^{2}}{1-\lambda \beta^{2} \rho}+\rho\right] \\
= & \frac{\gamma \sigma_{\varepsilon}^{2}}{\left(1-\rho^{2}\right)} \cdot \frac{\lambda \beta^{2}\left(1-\rho^{2}\right)+\rho}{1-\lambda \beta^{2} \rho} \\
= & \frac{\gamma \sigma_{\varepsilon}^{2}}{\left(1-\lambda \beta^{2} \rho\right)}\left[\lambda \beta^{2}+\frac{\rho}{1-\rho^{2}}\right] .
\end{aligned}
$$

Therefore, based on (G.5), (G.6) and (G.7),

$$
\begin{aligned}
\operatorname{Var}\left(\pi_{t}\right) & =\frac{1}{1-\lambda^{2} \beta^{4}}\left\{\lambda \beta^{2} \gamma \rho E\left[\left(\pi_{t}-\bar{\pi}\right)\left(y_{t}-\bar{y}\right)\right]+\gamma \rho E\left[\left(\pi_{t}-\bar{\pi}\right)\left(y_{t-1}-\bar{y}\right)\right]+\gamma^{2} \sigma_{\varepsilon}^{2}+\sigma_{u}^{2}\right\} \\
& =\frac{1}{1-\lambda^{2} \beta^{4}}\left\{\frac{\lambda \beta^{2} \gamma^{2} \rho \sigma_{\varepsilon}^{2}}{\left(1-\rho^{2}\right)\left(1-\lambda \beta^{2} \rho\right)}+\frac{\gamma^{2} \rho \sigma_{\varepsilon}^{2}}{\left(1-\lambda \beta^{2} \rho\right)}\left[\lambda \beta^{2}+\frac{\rho}{1-\rho^{2}}\right]+\gamma^{2} \sigma_{\varepsilon}^{2}+\sigma_{u}^{2}\right\} \\
& =\frac{\sigma_{\varepsilon}^{2}}{1-\lambda^{2} \beta^{4}}\left\{\frac{\gamma^{2} \rho\left[\lambda \beta^{2}\left(2-\rho^{2}\right)+\rho\right]}{\left(1-\rho^{2}\right)\left(1-\lambda \beta^{2} \rho\right)}+\gamma^{2}+\frac{\sigma_{u}^{2}}{\sigma_{\varepsilon}^{2}}\right\} \\
& =\frac{\sigma_{\varepsilon}^{2}}{1-\lambda^{2} \beta^{4}}\left\{\frac{\gamma^{2}\left(\lambda \beta^{2} \rho+1\right)}{\left(1-\rho^{2}\right)\left(1-\lambda \beta^{2} \rho\right)}+\frac{\sigma_{u}^{2}}{\sigma_{\varepsilon}^{2}}\right\} .
\end{aligned}
$$

According to (G.3),

$$
\begin{aligned}
E\left[\left(\pi_{t}-\bar{\pi}\right)\left(\pi_{t-1}-\bar{\pi}\right)\right] & =\lambda \beta^{2} \operatorname{Var}\left(\pi_{t}\right)+\gamma \rho E\left[\left(\pi_{t}-\bar{\pi}\right)\left(y_{t}-\bar{y}\right)\right] \\
& =\lambda \beta^{2} \operatorname{Var}\left(\pi_{t}\right)+\frac{\gamma^{2} \rho \sigma_{\varepsilon}^{2}}{\left(1-\rho^{2}\right)\left(1-\lambda \beta^{2} \rho\right)} .
\end{aligned}
$$

Thus, the correlation coefficient $\operatorname{Corr}\left(\pi_{t}, \pi_{t-1}\right)$ satisfies

$$
\begin{aligned}
\operatorname{Corr}\left(\pi_{t}, \pi_{t-1}\right) & =E\left[\left(\pi_{t}-\bar{\pi}\right)\left(\pi_{t-1}-\bar{\pi}\right)\right] / \operatorname{Var}\left(\pi_{t}\right) \\
& =\lambda \beta^{2}+\frac{\frac{\gamma^{2} \rho \sigma_{\varepsilon}^{2}}{\left(1-\rho^{2}\right)\left(1-\lambda \beta^{2} \rho\right)}}{\frac{\sigma_{\varepsilon}^{2}}{1-\lambda^{2} \beta^{4}}\left\{\frac{\gamma^{2}\left(\lambda \beta^{2} \rho+1\right)}{\left(1-\rho^{2}\right)\left(1-\lambda \beta^{2} \rho\right)}+\frac{\sigma_{u}^{2}}{\sigma_{\varepsilon}^{2}}\right\}} \\
& =\lambda \beta^{2}+\frac{\gamma^{2} \rho\left(1-\lambda^{2} \beta^{4}\right)}{\gamma^{2}\left(\lambda \beta^{2} \rho+1\right)+\left(1-\rho^{2}\right)\left(1-\lambda \beta^{2} \rho\right) \frac{\sigma_{u}^{2}}{\sigma_{\varepsilon}^{2}}} \\
& =\frac{\gamma^{2}\left(\lambda \beta^{2}+\rho\right)+\lambda \beta^{2}\left(1-\rho^{2}\right)\left(1-\lambda \beta^{2} \rho\right) \frac{\sigma_{u}^{2}}{\sigma_{\varepsilon}^{2}}}{\gamma^{2}\left(\lambda \beta^{2} \rho+1\right)+\left(1-\rho^{2}\right)\left(1-\lambda \beta^{2} \rho\right) \frac{\sigma_{u}^{2}}{\sigma_{\varepsilon}^{2}}}
\end{aligned}
$$




\section{H Proof of Proposition 4}

In order to check the conditions (B.1-B.2) of Section 6.2.1 in Evans and Honkapohja (2001, p.125), we rewrite the system in matrix form by

$$
X_{t}=A\left(\theta_{t-1}\right) X_{t-1}+B\left(\theta_{t-1}\right) W_{t},
$$

where $\theta_{t}^{\prime}=\left(\alpha_{t}, \beta_{t}, R_{t}\right), X_{t}^{\prime}=\left(1, \pi_{t}, \pi_{t-1}, y_{t}\right)$ and $W_{t}^{\prime}=\left(1, u_{t}, \varepsilon_{t}\right)$,

$$
\begin{aligned}
& A(\theta)=\left(\begin{array}{cccc}
0 & 0 & 0 & 0 \\
\lambda \alpha\left(1-\beta^{2}\right)+\gamma a & \lambda \beta^{2} & 0 & \gamma \rho \\
0 & 1 & 0 & 0 \\
a & 0 & 0 & \rho
\end{array}\right), \\
& B(\theta)=\left(\begin{array}{ccc}
1 & 0 & 0 \\
0 & 1 & \gamma \\
0 & 0 & 0 \\
0 & 0 & 1
\end{array}\right)
\end{aligned}
$$

As shown in Evans and Honkapohja (2001, p.186), $A(\theta)$ and $B(\theta)$ clearly satisfy the Lipschitz conditions and $B$ is bounded. Since $u_{t}$ and $\varepsilon_{t}$ are assumed to have bounded moments, condition (B.1) is satisfied. Furthermore, the eigenvalues of matrix $A(\theta)$ are 0 (double), $\rho$ and $\lambda \beta^{2}$. According to the assumption $|\beta| \leq 1,0 \leq \lambda<1$ and $0<\rho<1$, all eigenvalues of $A(\theta)$ are less than 1 in absolute value. Then it follows that there is a compact neighborhood including the SCEE solution $\left(\alpha^{*}, \beta^{*}\right)$ on which the condition that $|A(\theta)|$ is bounded strictly below 1 is satisfied.

Thus the technical conditions for Section 6.2.1 of Chapter 6 in Evans and Honkapohja (2001) are satisfied. Moreover, since $\pi_{t}$ is stationary under the condition $|\beta| \leq 1,0 \leq \lambda<$ 1 and $0<\rho<1$, then the limits

$$
\sigma^{2}:=\lim _{t \rightarrow \infty} E\left(\pi_{t}-\alpha\right)^{2}, \quad \sigma_{\pi \pi_{-1}}^{2}:=\lim _{t \rightarrow \infty} E\left(\pi_{t}-\alpha\right)\left(\pi_{t-1}-\alpha\right)
$$

exist and are finite. Hence according to Section 6.2.1 of Chapter 6 in Evans and Honkapohja (2001, p.126), the associated ODE is

$$
\left\{\begin{array}{l}
\frac{d \alpha}{d \tau}=\bar{\pi}(\alpha, \beta)-\alpha \\
\frac{d \beta}{d \tau}=\sigma^{-2}\left[\sigma_{\pi \pi_{-1}}^{2}-\beta \sigma^{2}\right] \\
\frac{d R}{d \tau}=\sigma^{2}-R
\end{array}\right.
$$


That is,

$$
\left\{\begin{array}{l}
\frac{d \alpha}{d \tau}=\frac{\lambda \alpha\left(1-\beta^{2}\right)+\gamma \bar{y}}{1-\lambda \beta^{2}}-\alpha=\frac{\alpha(\lambda-1)+\gamma \bar{y}}{1-\lambda \beta^{2}}, \\
\frac{d \beta}{d \tau}=\bar{F}(\beta)-\beta=\frac{\gamma^{2}\left(\lambda \beta^{2}+\rho\right)+\lambda \beta^{2}\left(1-\rho^{2}\right)\left(1-\lambda \beta^{2} \rho\right) \frac{\sigma_{u}^{2}}{\sigma_{\varepsilon}^{2}}}{\gamma^{2}\left(\lambda \beta^{2} \rho+1\right)+\left(1-\rho^{2}\right)\left(1-\lambda \beta^{2} \rho\right) \frac{\sigma_{u}^{2}}{\sigma_{\varepsilon}^{2}}}-\beta .
\end{array}\right.
$$

Hence a SCEE corresponds to a fixed point of the ODE (H.1). Furthermore, the SAC learning $\left(\alpha_{t}, \beta_{t}\right)$ converges to the stable $\operatorname{SCEE}\left(\alpha^{*}, \beta^{*}\right)$ as time $t$ tends to $\infty$.

\section{References}

[1] Adam, K., 2007. Experimental evidence on the persistence of output and inflation. Economic Journal 117, 603-636.

[2] Assenza, T., Heemeijer, P., Hommes, C. and Massaro, D., 2011. Individual Expectations and Aggregate Macro Behavior. CeNDEF Working Paper, University of Amsterdam.

[3] Berardi, M., 2007. Heterogeneity and misspecification in learning. Journal of Economic Dynamics \& Control 31, 3203-3227.

[4] Branch, W.A., 2004. The theory of rationally heterogeneous expectations: evidence from survey data on inflation expectations. Economic Journal 114, 592-621.

[5] Branch, W.A., 2006. Restricted perceptions equilibria and learning in macroeconomics, in: Colander, D. (Ed.), Post Walrasian Macroeconomics: Beyond the Dynamic Stochastic General Equilibrium Model. Cambridge University Press, New York, pp. 135-160.

[6] Branch, W.A., Evans, G.W., 2006. Intrinsic heterogeneity in expectation formation. Journal of Economic Theory, 127, 264-295.

[7] Branch, W.A., Evans, G.W., 2007. Model uncertainty and endogenous volatility. Review of Economic Dynamics 10, 207-237.

[8] Branch, W.A., McGough, B., 2005. Consistent expectations and misspecification in stochastic non-linear economies. Journal of Economic Dynamics \& Control 29, 659-676.

[9] Brock, W., Hommes, C., 1998. Heterogeneous beliefs and routes to chaos in a simple asset pricing model. Journal of Economic Dynamics \& Control 22, 1235-1274. 
[10] Bullard, J., 1994. Learning equilibria. Journal of Economic Theory 64, 468-485.

[11] Bullard, J., Evans, G.W., Honkapohja, S., 2008. Monetary policy, judgment and near-rational exuberance. American Economic Review 98, 1163-1177.

[12] Bullard, J., Evans, G.W., Honkapohja, S., 2010. A model of near-rational exuberance. Macroeconomic Dynamics 14, 166-188.

[13] Bullard, J., Duffy, J., 2001. Learning and excess volatility. Macroeconomic Dynamics 5, 272-302.

[14] Evans, G.W., Honkapohja, S., 2001. Learning and Expectations in Macroeconomics. Princeton University Press, Princeton.

[15] Fuhrer, J.C., 2006. Intrinsic and inherited inflation persistence. International Journal of Central Banking 2, 49-86.

[16] Fuhrer, J.C., 2009. Inflation persistence. Working paper.

[17] Fuster, A., Laibson, D. and Mendel, B., 2010. Natural expectations and macroeconomic fluctuations. Journal of Economic Perspectives 24, 6784.

[18] Gali, J., Gertler, M., López-Salido, J.D., 2001. European inflation dynamics. European Economic Review 45, 1237-1270.

[19] Guidolin, M., Timmermann, A., 2007. Properties of equilibrium asset prices under alternative learning schemes. Journal of Economic Dynamics \& Control 31, 161-217.

[20] Hamilton, J.D., 1994. Time Series Analysis. Princeton Univeristy Press.

[21] Heemeijer, P., Hommes, C.H., Sonnemans, J., Tuinstra, J., 2009. Price stability and volatility in markets with positive and negative expectations feedback. Journal of Economic Dynamics \& Control 33, 1052-1072.

[22] Hommes, C.H., Sonnemans, J., Tuinstra, J., van de Velden, H., 2005. Coordination of expectations in asset pricing experiments. Review of Financial Studies 18, 955-980.

[23] Hommes, C.H., 2011. The heterogeneous expectations hypothesis: some evidence for the lab. Journal of Economic Dynamics \& Control 35, 1-24. 
[24] Hommes, C.H., Sorger, G., 1998. Consistent expectations equilibria. Macroeconomic Dynamics 2, 287-321.

[25] Hommes, C.H., Rosser, J.B., 2001. Consistent expectations equilibria and complex dynamics in renewable resource markets. Macroeconomic Dynamics 5, 180-203.

[26] Hommes, C.H., Sorger, G., Wagener, F., 2004. Consistency of linear forecasts in a nonlinear stochastic economy. Unpublished working paper, University of Amsterdam.

[27] Lansing, K.J., 2009. Time-varing U.S. inflation dynamics and the new Keynesian Phillips curve. Review of Economic Dynamics 12, 304-326.

[28] Lansing, K.J., 2010. Rational and near-rational bubbles without drift. Economic Journal 120, 1149-1174.

[29] Lucas, R., 1972. Expectations and the neutrality of moeny. Journal of Economic Theory 4, 103-124.

[30] Marcet, A. and Sargent, T.J., 1989. Convergence of least squares learning mechanisms in self-referential linear stochastic models. Journal of Economic Theory 48, 337-368.

[31] Milani, F., 2007. Expectations, learning and macroeconomic persistence. Journal of Monetary Economics 54, 2065-2082.

[32] Muth, J.E., 1961. Rational expectaions and the theory of price movements. Econometrica 29, 315-335.

[33] Pfajfar, D., and B. Zakelj, 2010. Inflation Expectations and Monetary Policy Design: Evidence from the Laboratory. Mimeo, Tilburg University.

[34] Sargent, T.J., 1991. Equilibrium with signal extraction from endogenous variables. Journal of Economic Dynamics \& Control 15, 245-273.

[35] Sargent, T.J., 1993. Bounded Rationality in Macroeconomics. Oxford University Press Inc., New York.

[36] Sargent, T.J., 1999. The Conquest of American Inflation. Princeton University Press, Princeton, NJ.

[37] Sögner, L., Mitlöhner, H., 2002. Consistent expectations equilibria and learning in a stock market. Journal of Economic Dynamics \& Control 26, 171-185. 
[38] Tallman, E. W., 2003. Monetary policy and learning: some implications for policy and research. Federal Reserve Bank of Atlanta, ECONOMIC REVIEW, Third Quater 2003.

[39] Timmermann, A., 1993. How learning in financial markets generates excess volatility and predictability in stock prices. Quarterly Journal of Economics 108, 1135-1145.

[40] Timmermann, A., 1996. Excess volatility and predictability in stock prices in autoregressive dividend models with learning. Review of Economic Studies 63, 523-557.

[41] Tuinstra, J., 2003. Beliefs equilibria in an overlapping generations model. Journal of Economic Behavior \& Organization 50, 145-164.

[42] White, H., 1994. Estimation, Inference and Specification Analysis. Cambridge University Press, Cambridge.

[43] Woodford, M., 1990. Learning to believe in sunspots. Econometrica 58, 277307. 